NBER WORKING PAPER SERIES

NEURAL ACTIVITY REVEALS PREFERENCES WITHOUT CHOICES

\author{
Alec Smith \\ B. Douglas Bernheim \\ Colin Camerer \\ Antonio Rangel \\ Working Paper 19270 \\ http://www.nber.org/papers/w19270 \\ NATIONAL BUREAU OF ECONOMIC RESEARCH \\ 1050 Massachusetts Avenue \\ Cambridge, MA 02138 \\ July 2013
}

\begin{abstract}
Alec Smith would like to thank Todd Hare and Ian Krajbich for many helpful discussions. Financial support from NSF (BDB, CFC, AR), the Lipper Family Foundation (CFC) and the Betty and Gordon Moore Foundation (CFC) is greatly appreciated. The views expressed herein are those of the authors and do not necessarily reflect the views of the National Bureau of Economic Research.

At least one co-author has disclosed a financial relationship of potential relevance for this research. Further information is available online at http://www.nber.org/papers/w19270.ack

NBER working papers are circulated for discussion and comment purposes. They have not been peerreviewed or been subject to the review by the NBER Board of Directors that accompanies official NBER publications.
\end{abstract}

(C) 2013 by Alec Smith, B. Douglas Bernheim, Colin Camerer, and Antonio Rangel. All rights reserved. Short sections of text, not to exceed two paragraphs, may be quoted without explicit permission provided that full credit, including $(\mathcal{C}$ notice, is given to the source. 
Neural Activity Reveals Preferences Without Choices

Alec Smith, B. Douglas Bernheim, Colin Camerer, and Antonio Rangel

NBER Working Paper No. 19270

July 2013

JEL No. C91,D12

\begin{abstract}
We investigate the feasibility of inferring the choices people would make (if given the opportunity) based on their neural responses to the pertinent prospects when they are not engaged in actual decision making. The ability to make such inferences is of potential value when choice data are unavailable, or limited in ways that render standard methods of estimating choice mappings problematic. We formulate prediction models relating choices to "non-choice" neural responses and use them to predict out-of-sample choices for new items and for new groups of individuals. The predictions are sufficiently accurate to establish the feasibility of our approach.
\end{abstract}

Alec Smith

Division of the Humanities and Social Sciences

California Institute of Technology

Pasadena, CA 91125

alexandercharlessmith@gmail.com

B. Douglas Bernheim

Department of Economics

Stanford University

Stanford, CA 94305-6072

and NBER

bernheim@stanford.edu

\section{Colin Camerer}

Department of Economics

California Institute of Technology

Pasadena, CA 91125

camerer@hss.caltech.edu

Antonio Rangel

Department of Economics

California Institute of Technology

Pasadena, CA 91125

rangel@hss.caltech.edu

An online appendix is available at:

http://www.nber.org/data-appendix/w19270 


\section{Introduction}

A central problem in microeconomics is to predict the distribution of households' choices in not-yet-observed situations (e.g., after some policy intervention). The dominant tradition is to draw inferences from actual choices within some closely related domain. Unfortunately, that traditional approach often proves problematic due to various practical limitations of choice data: in some settings, data for closely related choices are either unavailable or extremely limited; the opportunity sets for naturally occurring choice problems are often impossible to characterize absent strong assumptions about expectations and other important considerations; and concerns about uncontrolled factors, selection, and the endogeneity of opportunity sets are endemic.

A sizable literature on stated preference (SP) techniques explores the feasibility of drawing reliable inferences from hypothetical choice data in contexts where actual choice data are either absent or deficient (for overviews, see Shogren, 2005, 2006). It is well established that answers to standard hypothetical questions are systematically biased, typically in the direction of overstating willingness-to-pay (WTP) and toward alternatives that are viewed as more virtuous. ${ }^{1}$ Two classes of solutions have been examined: one attempts to "fix" the hypothetical question; 2 the other seeks to correct for the bias through ex post statistical calibration. ${ }^{3}$ Because the limitations of those approaches are widely acknowledged, their use is largely confined to contexts where choice data pertaining to closely related decisions are entirely unavailable (e.g., in the environmental context, to value pristine coastlines, biodiversity, and the like), ${ }^{4}$ rather than merely deficient.

Despite the limitations of stated preference techniques, measures of elicited preferences remain potentially useful as long as it is possible to uncover stable predictive

\footnotetext{
${ }^{1}$ See, for example, Cummings et al. (1995), Johannesson et al. (1998), List and Gallet (2001), Little and Berrens (2004), Murphy et al. (2005), Blumenschein et al. (2007).

${ }^{2}$ Specific approaches include the use of (1) certainty scales as in Champ et al., (1997), (2) entreaties to behave as if the decisions were real (as in the "cheap-talk" protocol of Cummings and Taylor, 1999, or more recently the "solemn oath" protocol of Jacquemet et al., 2011), and (3) "dissonanceminimizing" protocols (as in Blamey et al., 1999, and Loomis et al., 1999, which allow respondents to express support for a public good while also indicating a low WTP).

${ }^{3}$ See Shogren (1993), Blackburn, Harrison, and Rutstrom (1994), Fox et al. (1998), List and Shogren $(1998,2002)$, and, to a lesser extent, Mansfield (1998). Ex post calibration (which can be traced to Kurz (1974), and was mandated by the National Oceanographic and Atmospheric Association, 1994), exploits a statistical relationship between real and hypothetical choices.

${ }^{4}$ In some cases, the object is to shed light on dimensions of preferences for which real choice data are unavailable by using real and hypothetical choice data in combination; see, e.g., Brownstone et al. (2000) and Small, Winston, and Yan (2005).
} 
relationships between them and real choices. Furthermore, since there may also be stable relationships between real choices and a much broader class of non-choice variables, there is no a priori reason to limit a prediction exercise to elicited preferences. Potential predictors include any reaction to elements of a contemplated opportunity set that occur when an individual is not engaged in actual decision-making (e.g., a subjective report or neural measurement assessed while imagining a consumption experience).

These observations suggest a more general strategy for predicting choices in situations where standard revealed preference methods are problematic: uncover the statistical relationships between real choices and combinations of non-choice variables, and use them (along with assessed values of the non-choice variables) to predict behavior out of sample in domains of interest. Because accurate forecasts of real choices "reveal preferences" in the classic sense of identifying what an individual would choose, we refer to this general class of procedures as non-choice revealed preference (NCRP). 5 Viewed from this perspective of this broader strategy, the historical success of the stated preferences approach may have been limited by its narrow focus on answers to hypothetical questions.

Potentially predictive non-choice measures fall into two broad categories: subjective reports (including but not limited to hypothetical choices), and physiological and neurobiological responses. An obvious virtue of relying on subjective reports is that the data are comparatively cheaper to collect. However, a rapidly growing body of work in psychology and neuroscience suggests that the biological measures might have predictive value-added over the subjective reports, probably because choices are systematically influenced by automatic processes that are not accessible to conscious awareness, but that

\footnotetext{
${ }^{5}$ We have repeatedly heard the following objection to the NCRP agenda (including all SP techniques): it involves out-of-sample predictions to domains for which actual choices may never be observed, and hence for which the stability of the predictive relationship cannot be verified. That objection is misguided. Out-of-sample prediction is commonplace in applied microeconomics; for example, it is present in any study that extrapolates an outcome (e.g., a policy effect) that is not directly observed. Whether an out-of-sample prediction can be verified is a function of the application, not of the method used to make the prediction (i.e., NCRP methods or traditional choicebased methods). Thus the objection is properly viewed not as a challenge to the NCRP approach per $s e$, but rather to the wisdom of particular applied agendas, such as valuing environmental damage by assessing the willingness-to-pay for a pristine environment. That said, we believe that even those applications are defensible. If a predictive relationship is shown to be stable over a sufficiently broad domain (including contexts related to the question of interest), and if a sufficiently large collection of out-of-sample predictions are successfully validated, then one can have reasonable confidence in the accuracy of out-of-sample predictions that cannot be validated. Those predictions in effect rely on identifying assumptions concerning the stability of the predictive relationship. As elsewhere in economics, one cannot test identifying restrictions, but one can make reasonable cases for them.
} 
can be measured with neurobiological techniques (see Dijksterhuis et al., 2006, Berns et al., 2010, Chua et al., 2011, and Falk et al., 2011). It is important to emphasize that the actual predictive power of non-choice responses is by no means evident without empirical confirmation, and hard to judge a priori without the type of systematic exercise carried out here.

This paper takes a first step in the development of NCRP methods that exploit nonchoice physiological responses: it evaluates the promise of those methods by investigating whether (and to what extent) non-choice neural responses measured using whole brain functional magnetic resonance imaging (fMRI) predict real choices. ${ }^{6}$ We recognize that the use of neural data raises issues of practicality, as its collection is likely to remain costly and inconvenient in the near term. In comparison, other physiological responses such as pupil dilation and skin conductance are easier to measure (and subjective responses are easier still). We nevertheless focus on whole brain fMRI measures because they provide a fairly comprehensive (albeit not perfect) picture of all the responses to a given stimulus, and thus are more likely to capture predictive information. By establishing the predictive power of such measures, we lay the foundation for subsequent efforts to identify the physiological manifestations of the predictive neural responses that are most easily and practically measured.

Because this first step is a substantial undertaking, the current paper does not fully execute the agenda articulated above. In particular, we make no attempt here to evaluate the incremental predictive power of neural data (over and above non-choice subjective reports), identify the most cost-effective physiological predictors, develop prediction models exploiting multiple varieties of non-choice variables, or bring the methods to practical applications. Nor have we exhausted all the possibilities for fine-tuning our methods to achieve the greatest possible predictive power from neural data (either at the image acquisition stage or the statistical analyses stage). These are tasks for ongoing and subsequent work.

Consistent with our objective of providing proof of concept, we confine attention to a narrow choice domain, consisting of choices among food items. Subjects "passively" view pictures of 100 snacks while undergoing an fMRI brain scan. After the passive scan is

\footnotetext{
${ }^{6}$ In other parallel work, some of us are currently evaluating the predictive power of a broad range of non-choice subjective reports. Our ultimate objective is to identify the combinations of non-choice responses - both subjective reports and physiological reactions - with the greatest predictive power.
} 
complete, they are unexpectedly asked to make choices among 50 pairs of snacks (one of which is implemented), with each snack appearing in one and only one pair. After completing the choices, they are asked to rate the extent to which they liked each item. Section III describes our experimental procedures in greater detail.

Our first objective is to construct, for each subject, a statistical model that predicts that subject's choices accurately out of sample (Section IV.A). Leaving out two pairs of items at a time, we estimate a prediction model based on the other 48 pairs, and use it to predict the individual's choice for the excluded pairs. Were we studying continuous choices, we would examine prediction bias, both overall and conditional on the values of the predictors. Because we are instead predicting dichotomous choices, we examine calibration, which is analogous to bias. A probability model is well-calibrated if predicted probabilities closely match actual choice frequencies. For example, events that are predicted to occur with $70 \%$ probability should actually occur $70 \%$ of the time. We evaluate calibration both overall and conditional on the values of the predictors.

A high degree of calibration would not, by itself, imply that non-choice neural responses are powerful predictors of choices. For example, a model that employs no predictors and assigns a $50 \%$ probability to the first of any two randomly ordered alternatives is perfectly calibrated. However, that model performs poorly with respect to resolution. Resolution is high when the predicted probability is close to $0 \%$ or to $100 \%$. A perfectly calibrated model with perfect resolution is ideal in the sense that it always predicts the outcome correctly.

Our focus on evaluating the calibration and resolution of probabilistic predictions is one of several important factors that distinguish this paper from the rest of the literature on the neural correlates of choice (see Section II for additional detail). A common practice in that literature is to report rates of successful classification ("success rates" for short). While we also report success rates (using probability $>50 \%$ as the classifier), we are of the view that the calibration and resolution of probabilistic forecasts are of greater interest to economists. A success rate reflects a particular blend of a model's calibration and resolution, one that provides a sufficient statistic for predictive performance under circumstances not commonly encountered in economics (e.g., where the object is to decide whether or not to treat a medical condition in a setting with a symmetric loss function). Knowing whether a success rate is high or low does not reveal whether one can use a model 
to construct accurate probability distributions for predicted choices, which is a paramount concern in economic applications.

For just over half of our subjects, we find that non-choice neural responses contain information that is useful in predicting choices, in the sense that our models significantly improve upon uninformed out-of-sample predictions (a 50\% success rate), usually with $\mathrm{p}<$ 0.01 . Moreover, even though there is no necessary relationship between success rates and calibration, we find that these models are on the whole well-calibrated out of sample. The difference between overall success rate $(68.2 \%)$ and the expected success rate $(72.9 \%)$ is modest. More significantly, across choice problems (within subject), there is a striking relationship between predicted probabilities and realized frequencies: a 10 percentage point increase in the former translates into roughly an 8 percentage point increase in the latter. Notably, that relationship is not driven by extreme cases (i.e., choice problems for which one item is universally and strongly preferred). While by no means perfect, this performance is, in our view, reasonably impressive in light of the task.

In contrast, for just under half of our subjects, success rates are low (below 60\%), and our models do not significantly improve upon uninformed predictions $(50 \%$ success rate), even at $\mathrm{p}<0.10$. Though low success rates do not necessarily imply poor calibration, these models also yield inaccurate out-of-sample probability statements. Thus, our procedure works well for just over half of our subjects, but not at all for the others.

Predicting choices on the level of a single individual is a demanding objective, one that goes beyond the requirements of most economic analyses, which are more typically concerned with aspects of group behavior - averages, aggregates, and possibly distributions. Group averages may be easier to predict, for example because various types of noise average out over multiple individuals. Accordingly, we next ask whether it is possible, for any particular group of individuals, to fit a model relating a measure of average subjective value to average non-choice neural responses for one set of items, and use that model successfully to predict the average subjective values of items not contained in the original set, based on the non-choice neural responses they induce (see Section IV.B). Compared with the successful half of our individual-level prediction exercises, our grouplevel prediction exercise achieves higher resolution and success rates, and the quality of calibration is comparable. We achieve this result despite including all subjects in the grouplevel analysis, regardless of whether their individual-level prediction exercises were successful. 
If non-choice neural activity exhibits a sufficiently similar relation to choice across subjects, then it should be possible to construct a single prediction model and use it without recalibration to predict choices based on neural measurements taken from new individuals or groups. Such a model would have considerable practical value in that, once estimated, it would vastly simplify the steps required to formulate additional predictions. To predict behavior in new situations, one could collect data on non-choice neural responses to the relevant prospects for a new group of individuals and apply an existing predictive model. It would not be necessary to gather the requisite data to estimate new predictive models for those subjects. Accordingly, we also investigate whether predictive models are portable across groups of individuals. We achieve a moderate degree of success when predicting a group's average valuation for new objects from a relationship estimated with data pertaining to other objects, gathered from another group.

Taken together, our results demonstrate that non-choice neural reactions to images of potentially desirable objects contain a great deal of information that can be used to predict decisions made by a particular individual, or average decisions made by a group of individuals, in new choice situations. Future improvements in methods and measurement technologies are likely to enhance the success of this approach.

\section{Related literature on the neurobiology of choice}

There is a substantial literature in neuroscience concerning the neural correlates of choice. With very few exceptions (discussed below), that literature is concerned with identifying neural activity that reliably encodes value signals during the act of choice; see, for example, Hsu et al., (2005), Kable \& Glimcher (2007), Knutson et al. (2007), Plassmann et al. (2007, 2010), Hare et al. (2008), and Levy et al. (2010). Consequently, the issues those studies address differ fundamentally from the ones that motivate our inquiry. Certainly, as Knutson et al. (2007) emphasize, it is possible to predict choices from neural activity measured during the act of decision-making. However, some economists take the view that there is little value in predicting choices in a setting where choices are themselves observable. If one's objective is to extrapolate choices based on neural activity in settings where choices are not observed, correlations between choice and choice-related neural reactions are not helpful (at least not directly).

Two recent studies suggest, however, that the brain's valuation circuitry may be active even when people are not actively engaged in choice. Lebreton et al. (2009) show 
that activity in the ventromedial prefrontal cortex (VmPFC) and the ventral striatum (vStr) while subjects were asked to judge the age of paintings, faces, and houses correlates with their subjective ratings for the same items (elicited in a separate task). Kang et al. (2011) show that activity in the vmPFC and the vStr correlates with the value of the stimuli during both real and hypothetical choices, which suggests that neural responses to real and hypothetical choices may share many common features. Thus, there is reason to hope that one can also reliably predict choices based on non-choice neural responses.

The current study is most closely related to recent neuroscience papers by Tusche et al. (2010) and Levy et al. (2011), both of which have elements of predicting choice (or tasks related to choice) from non-choice neural responses. ${ }^{7}$ To understand the key differences from our work, it is helpful to summarize several features of our analysis that are critical for the economic applications we envision. First, we are concerned with predicting real choices from neural responses during non-choice activity. Second, our interest is in out-of-sample prediction, rather than within-sample fit. We are concerned with predicting choices over one set of alternatives using a relationship estimated with data for a disjoint set of alternatives. ${ }^{8}$ Third, our objective is not merely to predict the more likely choice, but in addition to derive reliable probability statements concerning the alternatives. We seek a procedure that reliably indicates whether a particular alternative will be chosen with, say, $60 \%$ probability rather than $90 \%$ probability. Fourth, we are concerned with several distinct types of prediction exercises: within subject, within group, across subjects, and across groups. Predicting average behavior within and across groups likely has the greatest potential value for economics.

These four features distinguish our paper from the two studies listed above. None of them attempts to predict choices among one set of alternatives from a relationship estimated with a disjoint set of alternatives, nor do they attempt to derive and validate

\footnotetext{
${ }^{7}$ Our study is also related to Hampton and O'Doherty (2007), Grosenick et al. (2008), Krajbich et al. (2009), and Clithero et al. $(2009,2011)$. These papers employ the same class of methods from the statistical learning literature used here. However, in contrast to this paper, they do not try to predict out-of-scanner choices from non-choice neural activity. See also Haxby et al. (2001) for an early application of pattern classification techniques to fMRI data, and Pereira, Mitchell and Botvinick (2009), and Haynes (2011) for overviews of how methods from statistical learning are used more generally in brain imaging.

8 There is both an economic reason and a technical reason for this requirement. The economic reason is that we are ultimately concerned with predicting decisions for choice problems that are difficult or impossible to implement in practice. The technical reason, which we explain at the end of Section IV.A.1, is that statistical procedures might otherwise predict choices correctly by exploiting neural indicators of the alternatives' identities, rather than of their perceived values.
} 
probability statements concerning alternatives. Both studies focus exclusively on withinsubject classification or prediction, and they do not attempt to predict average behavior for groups, or choices across subjects. Tusche et al. (2010) study the neural correlates of hypothetical choices rather than real choices. Levy et al. (2011) predict real choices, but their subjects also made real decisions concerning the same objects during scanning, and hence their procedure does not truly involve non-choice neural responses in the sense defined here.

To be precise, the exercise in Levy et al. (2011) involves two phases: a localizer task and a neurometric prediction task. In the localizer task, subjects make decisions about whether or not to play lotteries. The goal of the task is to localize areas of the brain involved in valuation computations for each individual subject. In the prediction task, subjects are passively shown pictures of various types of goods (DVDs, CDs, stationary, monetary lotteries) and neural responses in the value areas identified in the localizer task are taken for each subject and good. After scanning, subjects make choices for every possible twoelement subset of the items, repeated twice. Several differences between their design and ours deserve emphasis. First, we predict choices on entirely new choice sets: in our task the choice pair consists of two new items, neither of which was used in fitting our model. Second, in Levy et al. subjects are also asked, every few trials, to make a purchase decision regarding the same stimuli used in the prediction task. Although these trials are not used in their neurometric prediction exercise, there is a concern that the mere presence of interspersed choice trials alters a subject's outlook on the items in question, so that they treat the passive trials more like actual purchase decisions. These differences between our task and that of Levy et al. are likely to make our exercise relatively more difficult than theirs. Finally, we employ a different prediction methodology that makes use of all the voxels in the brain rather than a specific region. In practice we are able to improve modestly upon the overall success rates achieved by Levy et al., despite the greater difficulty of the task.

The current paper also bears on the debate over the role of neuroeconomics within the broader field of economics. Various possibilities, such as the potential to develop useful neural tests of economic theories, remain controversial (see, for example, the contrasting views of Camerer, Loewenstein, and Prelec, 2004, Gul and Pesendorfer, 2008, and Bernheim, 2009). In contrast, it is hard to imagine even a traditionalist objecting to our agenda on the grounds that it is orthogonal to the goals of the field (in that our goals 
coincide with those of the stated preference method), or that it pursues those goals in a manner that is conceptually illegitimate (in that the task at hand is simply one of predicting an outcome using statistical models along with information that one could in principle collect). A skeptic might well question whether the method will prove useful in practice, but that is an empirical question, and hence one that should be resolved on the basis of evidence rather than prior belief.

\section{Experimental Design}

\section{A. Procedures}

Thirty-five right-handed subjects participated in the experiment (age range: 19 to 36 years old, 11 female). ${ }^{9}$ Subjects were pre-screened to ensure that they regularly ate the types of foods used in the experiment, and that they met the standard criteria required for the safe and reliable acquisition of fMRI data. Subjects were paid $\$ 100$ for participating, and were offered a $\$ 10$ bonus for limiting their head motion during the fMRI task (which, if excessive, invalidates the procedure). Despite these incentives, in-scanner head motion for eight subjects exceeded a pre-specified limit of $2 \mathrm{~mm}$ in any direction during a scanner run. After excluding those eight subjects, 27 usable subjects remained.

Subjects were instructed to refrain from eating or drinking anything other than water for four hours prior to the experiment. At the outset of a session, they were advised that the experiment would consist of three stages, and that they would receive the instructions for each stage only after completing the previous stage.10 Thus, as described below, when viewing images of snack foods in stage 1, subjects were not aware that they would subsequently face choices among pairs of those items in stage 2.

Stage 1. Passive viewing of foods during fMRI scan. In the first stage, subjects viewed images of 100 different snack foods while we measured their neural responses (see Figure A1 of Appendix A for sample images, and Table A1 of Appendix A for a list of all foods used in the experiment). Foods were shown in randomized order with each item appearing three times. Each image was visible for 2.75 seconds. Between images, a small white fixation cross centered on a black screen was shown for 8.25 seconds. For technical reasons related

\footnotetext{
${ }^{9}$ Subjects were recruited at Caltech, and the Caltech Institutional Review Board approved the experimental procedures.

10 A copy of the instructions is included in Appendix A.
} 
to the acquisition of the neural data, each session was divided into 6 identical blocks each consisting of 50 image presentations, separated by breaks of roughly one minute.

To enhance the psychological salience of the images, we told subjects that they would be required to eat at least three spoonfuls of one of the food items at the end of the session. With $50 \%$ probability, the item would be selected at random, and with 50\% probability it would be determined in a subsequent stage of the experiment. However, subjects were not told that that they would be asked to make choices among the alternatives.

Given the tedious nature of the task, we inserted five additional "catch" trials at random intervals within every block. During each such trial, the subject was instructed to press a button indicating whether the displayed item was sweet or salty. Subjects were given a maximum of 2.75 seconds to respond, after which a fixation cross screen appeared for 8.25 seconds. The foods shown in the catch trials were different from those used in the passive viewing trials, and we did not use the neural responses from the catch trials in the prediction exercises described below. In $93.1 \%$ of the catch trials, subjects responded within the time allowed, which suggests that they attended to the images. ${ }^{11}$

We collected measures of neural activity using BOLD-fMRI (blood-oxygenated level dependent functional magnetic resonance imaging). ${ }^{12}$ Instead of making assumptions as to which brain regions were likely to generate predictive non-choice responses, we measured activity throughout the entire brain, and used all of the data in our prediction exercises. It is natural to conjecture that brain regions previously shown to be involved in valuation, such as the medial prefrontal cortex or the ventral striatum, ${ }^{13}$ will play critical roles in predicting choices. However, we decided to carry out our prediction exercise using the entire set of brain responses for three reasons. First, we wanted to demonstrate that the NCRP methodology proposed here does not depend on knowledge of which brain circuits are

\footnotetext{
${ }^{11}$ For one subject, we did not observe any responses to catch trials during the last two blocks. This subject is included in our analyses, but excluding him does not affect our results substantially. 12 The fMRI data were collected at the Caltech Brain Imaging Center using a Siemens 3T Trio scanner. We acquired gradient-echo T2* weighted echo planar (EPI) images with BOLD contrast. We used an oblique acquisition angle of 30 degrees relative to the anterior commissure-posterior commissure line (Deichmann et al., 2003) and an 8-channel phased array head coil to maximize functional contrast-to-noise in areas of the ventromedial prefrontal cortex which, as described in Section II, have been shown to play a critical role in valuation. Each volume consisted of 44 axial slices covering the entire brain. The imaging parameters were: echo time, $30 \mathrm{~ms}$; field of view, $192 \mathrm{~mm}$; in-plane resolution and slice thickness, $3 \mathrm{~mm}$; repetition time (TR), $2.75 \mathrm{~s}$.

${ }^{13}$ For a review of the literature see Rangel and Hare (2010).
} 
involved in the choice process, or how to measure their activity. Second, the usefulness of a brain region for our predictive task depends on: 1) how cleanly we can measure neural activity in the region; 2) how well that activity correlates with automatic valuations; and 3) how much predictive information the activity in that region adds over and above other activity used to construct the predictions. We use data from the whole brain to allow for the possibility that neural activity in some brain regions will prove informative after accounting for neural activity in other regions. This is particularly important, because the signal-tonoise of BOLD-fMRI in areas typically associated with valuation (like ventral striatum and ventromedial prefrontal cortex) is relatively low. In fact, the accuracy of our method declines when we restrict attention solely to the ventral striatum and ventromedial prefrontal cortex, indicating the value of our whole-brain approach. Third, many psychological processes exhibit some correlation with value, such as attention and arousal. This implies that many voxels, besides those in areas known to be involved in valuation, will also correlate with values, and would have independent noise (Litt et al., 2011). These independent measurements are useful in prediction.

BOLD-fMRI operates by measuring changes in local magnetic fields resulting from local inflows of oxygenated hemoglobin and outflows of de-oxygenated hemoglobin that occur when neurons fire. The BOLD signal is correlated with aggregate neural activity within relatively small "neighborhoods" (tiny cubes, known as voxels). One complication is that BOLD responses are slower than the associated neuronal responses: although the bulk of the neuronal response takes place in 4 to 6 seconds, subsequent BOLD measurements are affected for as much as 24 seconds. Even so, as long as trials are spaced sufficiently far apart, one can attribute most of the BOLD signal to trial-specific neural responses. In our experiment, each trial spanned a total of 11 seconds (2.75 seconds for an image, and 8.25 seconds for a fixation cross on a black screen), and BOLD measurements were obtained in $3-\mathrm{mm}^{3}$ voxels every 2.75 seconds. With this design, the BOLD signal provides a good measure of local neural responses to each image. This is an approximation, but it suffices for our purposes. Presumably, a sharper measure of neural activity would yield even greater predictive power than that of the somewhat noisy measure used here.

Stage 2: Pairwise choices. In the second stage of the experiment, subjects were shown pairs of food items outside the scanner, and were asked to choose their preferred item from each pair. They were told that, with $50 \%$ probability, one of the pairs would be selected at random, and they would receive their choice from that pair. 
The first ten subjects were shown 200 pairs of items drawn randomly with replacement from the 100 foods viewed in stage 1 . The remaining 17 subjects were shown 50 randomly selected pairs, with each item appearing in a single pair. As discussed below, the first procedure is not appropriate for some portions of our analysis (a fact which we did not realize until we examined some preliminary results). Accordingly, some of the results reported below are based on all 27 subjects, while others are based on the last 17.

Foods were randomly assigned to left and right positions on the screen. As is common in such tasks, there was a small spatial bias: subjects chose the left item $53 \%$ of the time ( $p<0.05$, binomial test). When estimating our forecasting models, it is important to ensure that our predictions do not benefit artificially from this bias (as they would if we used models describing the probability of choosing the object displayed on the left). Accordingly, for every subject, we randomly divided the choice pairs into two equal sets: in one, the chosen item was designated as the "target," while in the other the item not chosen was so designated. The choice for any trial was then coded as a 1 if the target item was chosen, and as a 0 otherwise. With this assignment, the unconditional probability that our discrete choice variable equals 1 in any given trial is exactly 50 percent, and the predictive success of more elaborate models must be judged against that neutral benchmark (rather than 53 percent). ${ }^{14}$

Stage 3: Preference ratings. In the final stage of the experiment, subjects were asked to indicate the extent to which they liked each food item, using a discrete scale from -3 (strongly dislike) to 3 (strongly like). They viewed pictures of all 100 items sequentially and entered liking ratings through button presses, proceeding at their own pace. They were told that their ratings would not affect the item they received at the end of stage 3 , but they were also strongly encouraged to provide ratings that reflected their true preferences.

After each subject finished rating the items, we tossed a coin to determine whether he or she would receive an item chosen at random, or the item chosen in a randomly selected choice trial from stage 2 (where the item or choice trial was selected by drawing a number from an envelope). Subjects were required to eat at least three spoonfuls of the selected item, and were allowed to eat more if desired. Subjects were instructed to remain in the lab for 30 minutes, during which time they were not permitted to eat anything else.

\footnotetext{
${ }^{14}$ Note that because the target item is designated at random, spatial bias effectively introduces random variation into the discrete choice variable that is inherently not predictable from stage 1 measurements. Thus, spatial bias necessarily reduces the predictive accuracy of our models.
} 


\section{B. Initial data processing}

Before analyzing the predictive power of non-choice BOLD responses, the raw data must be converted into a usable form. First, we corrected for head motion to ensure that the time series of BOLD measurements recorded at a specific spatial location within the scanner is always associated with the same brain location throughout the experiment. ${ }^{15}$ Second, we removed low-frequency signals that are unlikely to be associated with neuronal responses to individual trials. ${ }^{16}$ Third, we realigned the BOLD responses for each individual into a common neuroanatomical frame (the standard Montreal Neurological Institute EPI template). This step, called spatial normalization, is necessary because brains come in different shapes and sizes, and as a result a given spatial location maps to different brain regions in different subjects. Spatial normalization involves a non-linear re-shaping of the brain to maximize the match with a target template. Although the transformed data are not perfectly aligned across subjects due to remaining neuroanatomical heterogeneity, the process suffices for the purposes of this study. Any imperfections in the re-alignment process introduce noise that reduces our ability to predict choices.

For the analyses described in Sections V (which involve comparisons across subjects), we also spatially smoothed the BOLD data for each subject, by making BOLD responses for each voxel a weighted sum of the responses in neighboring voxels, with the weights decreasing with distance. ${ }^{17}$ This transformation addresses residual problems arising from neuroanatomical heterogeneity across subjects. In effect, smoothing assumes that any particular voxel in one subject's brain can play the same predictive role as neighboring voxels in another subject's brain; without smoothing, we would be assuming that only the same voxel can play the same predictive role.

The final step was to compute, for each subject and each voxel, the average nonchoice neural response to each food item. We began by removing predicted neural responses that were related to the task (e.g., seeing the image of a food item) but common

\footnotetext{
15 BOLD measurements were corrected for head motion by aligning them to the first full brain scan and normalizing to the Montreal Neurological Institute's EPI template. This entails estimating a sixparameter model of the head motion ( 3 parameters for center movement, and 3 parameters for rotation) for each volume, and then removing the motion using these parameters. For details, see Friston et al. (1996).

${ }^{16}$ Specifically, we applied a high-pass temporal filter to the BOLD data with a cut-off of 128 seconds.

${ }^{17}$ Smoothing was performed using an $8 \mathrm{~mm}$ full-width half-maximum Gaussian kernel.
} 
to all items. ${ }^{18}$ The object of this step is to restrict attention to BOLD responses that are specific to individual food items, and therefore likely to be helpful in predicting choices. Second, we averaged the residual response over the three presentations of each food item, collected 2.75 and 5.5 seconds after the onset of stimulus. In constructing this average, we omitted measurements from full brain scans (known as volumes) that exhibited excessive within-volume variation across voxels. ${ }^{19}$ This exclusion criterion reduces noise (and thereby improves predictive accuracy) by eliminating signals that are outliers with respect to the typical range of BOLD responses for food items.

\section{Predicting choices involving new items, within subjects and groups}

The canonical task motivating our investigation is to determine how people will behave when confronted with some new or difficult-to-observe choice situations. Imagine assembling a group of individuals, measuring their non-choice neural responses to prospects that we can actually implement, as well as to the new choice situations, and then presenting them with unanticipated choices among the implementable prospects. We can then estimate the relationship between their choices and non-choice responses for the implementable prospects, and use that relationship along with non-choice neural responses for the new situations to predict behavior in those situations. Do the non-choice neural responses contain enough information to make reasonably accurate predictions?

In this section, we implement the procedure outlined in the previous paragraph and use it to make and evaluate predictions both within subjects and within groups.

\section{A. Within-subject predictions}

\footnotetext{
18 We carried out this step by estimating a general linear model (GLM) of BOLD responses with an AR(1) structure. The model included the following regressors: an indicator function for the moment at which the image of an item appears on the screen, convolved with a canonical hemodynamic responses function (Friston et al., 1998) that captures the manner in which neural responses are mapped to delayed changes in the BOLD signal, six block dummies, and the time series of head motion parameters estimated in the pre-processing step described above. The residuals from this regression capture the BOLD responses from each trial that are item-specific. For reasons of practicality, we performed this calculation only for gray-matter voxels (of which there are approximately 45,000 per-subject). We identified gray matter in each subject using the Automated Anatomical Labeling (AAL) Tool and the MNI gray-matter mask (Tzourio-Mazoyer et al 2002).

${ }^{19}$ For each volume we computed the variance across voxels (known as global signal variation), as well as the mean and standard deviation of this variance across volumes. We excluded data on volumes for which the global signal variation exceeded the median plus five mean absolute deviations.
} 
In this subsection, we focus on the accuracy of within-subject predictions. For reasons detailed below, we restrict attention to subjects 11 through 27, each of whom made decisions for 50 pairs of food items, with no item appearing twice.

Statistical methods. We adopt a logit probability model for choices. For every subject $i$ and choice pair $t$, let $y_{i t}=1$ if the target food was chosen, and $y_{i t}=0$ otherwise. ${ }^{20}$ For every brain voxel $v$ and choice pair $t$, let $d_{i t v}$ denote the difference between the measured neural responses in voxel $v$ to the target and non-target food items offered in choice pair $t$ (i.e., the response for the target food minus the response for the non-target food). Also let $D_{i t}$ denote the vector of differential neural responses for all voxels. The following probability statement describes our model:

$$
\operatorname{Pr}\left(y_{i t}=1 \mid D_{i t}\right)=\frac{\exp \left(\gamma_{0}+\gamma D_{i t}\right)}{1+\exp \left(\gamma_{0}+\gamma D_{i t}\right)} .
$$

Because our object is accurate out-of-sample prediction, we employ standard methods for estimating and evaluating predictive models. A central tenet of the prediction literature is that within-sample fit is a poor gauge of out-of-sample fit (cf. Efron, 1986). Therefore, we employ cross-validation (Stone, 1974) for both model assessment and model selection. Typically, one proceeds by dividing the sample into a "training sample" which is used for estimation, and a "hold-out" sample that is used to evaluate predictions. By removing two choice pairs at a time from the set of 50, we create 25 training samples (each consisting of 48 observations) and 25 associated hold-out samples (each consisting of 2 observations). For each training sample, we estimate the model and use it to predict choices for the associated hold-out observations. We then assess the model's out-of-sample predictive performance over all 25 hold-out samples (50 predictions in all).

To ensure the representativeness of both the training and hold-out samples, we randomly partitioned the 50 choices into 25 pairs, with each pair containing one choice from which the target item was chosen, and another from which it was not chosen. Each such pair served as a hold-out sample, and the complement served as a training sample. This procedure, known as stratified cross-validation, yields training and hold-out samples in which the target item is chosen exactly 50 percent of the time, just as in the full sample (by construction). Leave-one-out cross-validation is an approximately unbiased method for estimating the true (expected) prediction error (Hastie et. al, 2009, page 242). However, leave-one-out cross validation estimators may have high variance, and simulation studies

${ }^{20}$ As described in the previous section, one item in every pair was randomly designated as the target. 
have concluded that stratified cross-validation has better performance in terms of both bias and variance than regular cross-validation (Kohavi, 1995). Because our samples are unbalanced with leave-one-out cross validation, we compromise and employ stratified leave-two-out cross validation.

As the literature recognizes, evaluating the predictive performance of a categorical probability model involves some inherent ambiguities. Alternative standards for defining a "predicted outcome" have been proposed. In the context of binary models, Cramer (1999) proposes identifying an alternative as the predicted outcome if its predicted probability exceeds its baseline frequency in the population. ${ }^{21}$ By construction, in our experiment, the baseline frequency for selecting the target item is exactly $50 \%$ for each subject. Consequently, we classify the target item as the predicted choice if its predicted probability exceeds 50\%; otherwise, the non-target item is the predicted choice. We classify a particular prediction as a "success" if the predicted item was in fact chosen.

Notice that our task involves prediction from small samples (48 observations). It therefore raises two important and closely related issues: model selection and overfitting.

The model selection problem is obvious: because we estimate each model using only 48 observations, we cannot use all 45,000 potential predictors (voxel-specific BOLD signals). ${ }^{22}$ Instead we must focus on a small handful of predictors, in effect leaving out large numbers of presumably relevant variables. If we intended to interpret estimated parameters as reflecting causal effects, the left-out variable problem would be fatal. Accordingly, it is essential to emphasize that our objective here is purely prediction. When predicting from a small sample, it is worthwhile to include a variable only if the incremental predictive information it carries is sufficient to justify sacrificing a scarce degree of freedom. Thus, for example, when two important causal factors are highly correlated, it is often appropriate to include only one, because each reflects most of the predictive information contained in the other. Naturally, with either factor omitted, the coefficient of the included factor will not measure its causal effect; on the contrary, that coefficient will also reflect the causal effect of the omitted factor. Even so, the omission of a causal factor does not impart a bias to predictions (conditional on the included predictors), and may well reduce variance. Statistical tools for model selection include the Akaike Information Criterion (AIC), the

\footnotetext{
${ }^{21}$ Even that alternative is recognized as somewhat arbitrary; see Green (2003), p. 685.

22 See Chapter 18 of Hastie et al. (2009) for an overview of statistical techniques for prediction problems when the predictors greatly outnumber the observations. Within the economics literature, see also Fan, Lv, and Qi (2011), and Belloni, Chernozhukov, and Hansen (2011).
} 
Bayesian Information Criterion (BIC), cross-validated predictive performance, LASSO (which we describe and implement below), and others.

The overfitting problem arises because, with small samples (and especially with many predictors), there is a substantial likelihood that some predictor will be highly correlated (within sample) with the outcome variable purely by chance. While OLS estimates will still yield statistically unbiased predictions, the variance of the prediction error can be extremely high, and hence predictions can prove inaccurate. The most obvious case occurs when the number of predictors equals the number of observations. In our analysis, any combination of 48 linearly independent predictors will yield a perfect fit within sample, but the resulting model will generally perform very poorly out of sample.

Various techniques have been developed to address the overfitting problem. Shrinkage estimators (of which ridge regression is the best known example) compensate for overfitting by shrinking the overall size of the estimated coefficient vector. Such estimators can attenuate the sensitivity of predictions to changes in the predictors, and hence reduce variance, thereby improving the overall accuracy of out-of-sample predictive performance according to measures such as mean-squared prediction error (a commonly used statistic that encompasses both the bias and the precision of a prediction). We address the model selection and overfitting issues using LASSO (the Least Absolute Shrinkage and Selection Operator; see Tibshirani, 1996) combined with cross-validation. As the name implies, LASSO, like ridge regression, is a shrinkage procedure. ${ }^{23}$ For both procedures, one optimizes a standard criterion for within-sample fit (for example, minimizing the sum of squared residuals in the case of a regression, or maximizing likelihood) subject to a penalty that increases monotonically in the size of the coefficient vector. For ridge regression, one measures the size of the coefficient vector using the $\mathrm{L}_{2}$-norm (i.e., the square root of the sum of squared coefficients). For LASSO, one uses the $\mathrm{L}_{1}$-norm (i.e., the sum of the absolute values of the coefficients). While both methods of penalization lead to shrinkage, only LASSO also accomplishes variable selection. ${ }^{24}$

\footnotetext{
23 In a linear regression context, one can also interpret LASSO as a Bayesian regression with double exponential priors; see Tibshirani (1996). In the Bayesian context, shrinkage results from the priors. 24 Relative to an $\mathrm{L}_{2}$-penalty, an $\mathrm{L}_{1}$-penalty favors coefficient vectors wherein some elements equal zero. Notice, for example, that in a model with two coefficients, $\gamma_{1}$ and $\gamma_{2}$, as we move linearly from $\left(\gamma_{1}, \gamma_{2}\right)=(\alpha, 0)$ to $\left(\gamma_{1}, \gamma_{2}\right)=(\alpha / 2, \alpha / 2)$, the $L_{1}$-penalty remains constant while the $\mathrm{L}_{2}$-penalty declines monotonically. More importantly, because iso-penalty curves are smooth when one uses the $\mathrm{L}_{2}$-norm, solutions involve coefficients of zero only by coincidence. In contrast, because iso-penalty curves are
} 
In our context, the LASSO procedure involves maximizing the following penalized log-likelihood function over the parameters $\gamma_{0}$ and $\gamma=\left(\gamma_{1}, \ldots, \gamma_{v_{i}}\right)$, where $v_{i}$ is the number of voxels for subject $i$ :

$$
\frac{1}{T} \sum_{t=1}^{T}\left\{y_{i t} \log p_{i t}+\left(1-y_{i t}\right) \log \left(1-p_{i t}\right)\right\}-\lambda\|\gamma\|_{1} .
$$

Here, $T$ denotes the number of trials in the training set, $p_{i t}=\operatorname{Pr}\left[y_{i t}=1 \mid D_{i t}\right]$ and $\|\gamma\|_{1}$ denotes the $\mathrm{L}_{1}$ norm on $\gamma^{25}$ In the LASSO objective function, the $\mathrm{L}_{1}$ penalty receives a weight of $\lambda .{ }^{26}$ Larger values of $\lambda$ lead to greater shrinkage and to more aggressive variable selection. The value of $\lambda$ is determined through cross-validation, which is a procedure for simulating out-of-sample predictive performance entirely within a training sample. For our analysis, we randomly divided each training sample into five sets of approximately equal size, indexed $k=1, \ldots, 5$ (called folds in the statistical prediction and machine learning literatures). For each $k$, we estimated the penalized regression model for each possible value of $\lambda$ in a pre-specified grid using only the data from the $k-1$ other folds. We then used the estimated models to predict choices for the left-out fold, and computed the accuracy of the predictions by comparing them to the actual choices. The value of $\lambda$ with the highest successful prediction rate across all of the folds, $\lambda^{*}$, was then used to estimate the model with all of the observations in the training sample. ${ }^{27}$ Importantly, note that the selection of $\lambda^{*}$ is blind with respect to outcomes in the actual hold-out sample; thus, accuracy within the hold-out samples remains a valid gauge of the procedure's out-of-sample performance.

The LASSO procedure not only achieves beneficial shrinkage, but also in effect ensures that a variable remains in the model with a non-zero coefficient only if its incremental predictive value is sufficient to justify the sacrifice of a degree of freedom. Thus, in our setting the procedure selects the brain voxels with the neural responses that provide the most valuable predictive informative concerning subsequent choices.

Prior to implementing the LASSO procedure, we reduced the vast set of candidate voxels by excluding those that failed to meet a simple statistical criterion. Ryali et al. (2010)

kinked at the axes when one uses the $\mathrm{L}_{1}$-norm, solutions typically involve setting many coefficients equal to zero.

25 Note that the probabilities $p_{i t}$ depend on $\gamma_{0}$, but that this term is not penalized in the LASSO specification.

26 To fit the model we used the glmnet software package for MATLAB (Friedman, Hastie, \& Tibshirani 2010).

27 We use the out-of sample prediction (success) rate here and throughout this paper as our criteria for selecting $\lambda^{*}$, in order to maximize predictive success. An alternative criterion is log-likelihood. 
have shown that this initial screening step can improve predictive accuracy in studies employing fMRI data, even when the subsequent estimation procedure selects voxels automatically (as is the case here). For every voxel, we computed a simple two-sided t-test for the hypothesis of no difference between neural responses (within the training sample) to foods that were chosen and those that were not. We then ranked voxels by the absolute values of their t-statistics, and retained only those exceeding some threshold percentile. ${ }^{28}$ Intuitively, the purpose of this initial screening step is to focus attention on voxels that are likely to contain highly predictive information. For each prediction task, we examine the robustness of prediction success rates with respect to a range of screening criteria, and then present more detailed results based on analyses for which the top $1 \%$ of voxels were retained. Note that the voxel selection procedure, like the selection of $\lambda^{*}$, is completely blind with respect to outcomes in the actual hold-out sample; thus, accuracy within the hold-out samples remains a valid gauge of the procedure's out-of-sample performance.

As we mentioned at the outset of this section, data gathered from our first 10 subjects were not used for within-subject predictions. Recall that those subjects made choices from 200 pairs of items, drawn randomly with replacement from our set of 100 items. Thus, when the full sample is divided into a training sample and a hold-out sample, the items that belong to pairs in the hold-out sample also typically belong to pairs in the training sample. The resulting overlap between the sets of items represented in the training and hold-out samples can lead to spurious predictive accuracy. ${ }^{29}$

\footnotetext{
${ }^{28}$ According to Ryali et al. (2010), this screening step can improve predictive performance in these settings.

${ }^{29}$ To see why, suppose for the purpose of illustration that the subject's choices are pair-wise transitive. From the choices in the training sample, one can then predict many choices perfectly out of sample. For example, if the individual chooses $a$ over $b$ as well as $b$ over $c$ in the training sample, we can confidently predict that he will pick $a$ over $c$ out of sample; no neural information is required. This observation is problematic because, with 45,000 voxels, there is a substantial likelihood that each item will be associated with some voxel within which neural activity was high when the item in question, and only that item, was presented. That voxel serves as a spurious neural identifier for the item. LASSO tends to retain those voxels and assign them coefficients that reflect each item's place in the subject's preference ordering. In our example, it might assign coefficients of 1,0 , and -1 to the voxels identifying, respectively, items $a, b$, and $c$. Accordingly, the resulting model will predict that $a$ will be chosen over $c$ out of sample, but only because the neural activity spuriously identifies the item, and not because it is correlated with some provisional assessment of subjective value. We discovered this problem after collecting data on the first 10 subjects and obtaining results indicating a degree of predictive accuracy that seemed too good to be true (i.e., well in excess of $80 \%$ ). Subsequently, we avoided the problem by selecting the choice pairs for subjects 11 through 27 so that each item appeared in one and only one pair. We include the data gathered from the first ten subjects only in the analyses of Sections IV.B and V, where the problem does not arise.
} 
Results. Figure 1 plots the mean success rates, defined as the fraction of hold-out observations for which the predicted item was chosen, as a function of the percent of voxels retained after initial screening, with the retained percentiles ranging from $0.01 \%$ to $100 \%$. When $1 \%$ of voxels are retained, the mean success rate is $61.3 \%$, which represents an economically and statistically significant improvement over the uninformed 50\% benchmark ( $p<0.0001$, one-sided t-test). Performance falls sharply when fewer than $1 \%$ of voxels are retained, but declines only slightly when fewer are eliminated. Indeed, when we abandon the initial screening step (i.e., retain all voxels), our overall success rate, 59.3\%, remains significantly better than the uninformed benchmark $(p<0.001)$, and is not significantly different from the rate obtained when retaining $1 \%$ of voxels $(\mathrm{p}=0.23$, paired $\mathrm{t}$ test). Thus we find, in contrast to Ryali et al. (2010), that the initial voxel-screening step yields only a small and statistically insignificant improvement in this measure of predictive accuracy. For the rest of this section, we will focus on the results obtained using the $1 \%$ screening criterion; our conclusions are not substantially affected by applying less restrictive screens.

The first data column in Table 1 provides results on success rates for each subject (numbered 11 through 27 because this analysis excludes the first ten subjects). There was considerable cross-subject variation in success rates, which ranged from a low of $44 \%$ to a high of $76 \%$, with all but one exceeding $50 \%$ and four exceeding $70 \%$. These rates exceeded the uninformed benchmark by a statistically significant margin for 9 out of 17 subjects at the 5\% level (amongst whom the overall success rate was 68\%), and for 8 out of 17 subjects at the $1 \%$ level. Plainly, non-choice neural responses contain a substantial amount of predictive information for those subjects. For subsequent reference, we have shaded all of the rows in the table associated with high-success-rate subjects (i.e., those whose success rates exceeded the uninformed benchmark by statistically significant margins), so that their results are easily distinguished from those of low-success-rate subjects (i.e., the complementary set).

As we mentioned in the introduction, a success rate is a particular blend of the resolution and calibration of a probabilistic prediction..$^{30}$ Resolution refers to the degree of certainty. The statement that an individual will choose the target item with either $1 \%$ or

\footnotetext{
${ }^{30}$ These terms refer to a decomposition of the mean squared forecast error or Brier score (Brier 1950). Our discussion follows Murphy's (1973) decomposition; see also Yates (1982), and Murphy \& Winkler (1987).
} 
99\% probability involves high resolution, while the statement that he will choose that item with either $49 \%$ or $51 \%$ probability involves low resolution. Calibration refers to the degree to which the probabilistic prediction matches actual frequencies. To illustrate, suppose that for some group of pairwise choices, a model predicts that the target will be chosen with an average probability of $75 \%$. The model's predictions are well-calibrated if the realized frequency that the target is chosen, for any reasonably large group of observations, is close to the average predicted probability. If it is not close, the model's predictions are poorly calibrated. In the preceding example, if the realized frequency is 55\% rather than $75 \%$, the model's probabilistic predictions are poorly calibrated. The same is true if the frequency is $95 \%$.

According to these definitions, the predictions of the uninformed benchmark (5050) have zero resolution but are perfectly calibrated (because the overall success rate, $50 \%$, matches the predicted probability of the most likely item in every pair). In contrast, the typical deterministic model will be highly resolved, but in all likelihood poorly calibrated (because it is rarely possible to forecast outcomes with certainty).

Knowing only that the average success rate for our procedure is $61.3 \%$, one cannot say anything about the resolution or calibration of the underlying predictions. Yet such distinctions are plainly crucial. If our procedure typically yielded predicted probabilities of the more likely item on the order of $90 \%$ but achieved an overall success rate near $60 \%$, its success would be only directional, and one would not be able to take its probabilistic predictions seriously. On the other hand, if on average our procedure yielded predicted probabilities of the more likely item near $60 \%$ (i.e., in line with the observed success rate), then although one could complain that its predictions had somewhat low resolution, at least they would be well-calibrated. ${ }^{31}$

With respect to potential complaints concerning low resolution, it bears emphasizing that the value of an accurate predictive model should not be discounted merely because its predictions are not as highly resolved as one might like. On the individual level, certain determinants of choice may be fundamentally unpredictable (see, e.g., Krajbich, Armel, and Rangel, 2010), in which case the resolution of any well-calibrated probabilistic prediction is necessarily limited. Fortunately, such idiosyncratic randomness

\footnotetext{
${ }^{31}$ As explained below, further investigation would be required before reaching that conclusion.
} 
likely averages out over multiple decisions, so it should still be possible to predict the average behavior of groups with reasonably high resolution (see Sections IV.B and V).

The second data column in Table 1 sheds light on the resolution of our procedure's predictions. Focusing for the moment on the second-to-last row, we see that the mean predicted probability of the more likely item is $72.7 \%$. For a perfectly calibrated model, this number equals the expected success rate. Yet we see that there is a sizable and highly statistically significant gap (or bias) of 11.4 percentage points between the mean predicted probability and the overall success rate of $61.3 \%(p<0.001)$. At this level of aggregation, one cannot describe the models' probabilistic predictions as well-calibrated.

A careful examination of the results for individual subjects tells a more interesting and nuanced story. Based on our initial analysis of success rates for individual subjects, it is entirely possible that our procedure works well for some subjects, and poorly (or not at all) for others. For example, some subjects may not meaningfully attend to the images of food items during stage 1.32

To evaluate the calibration of the predictive model for each subject, we first test the hypothesis that the success rate equals the mean predicted probability of the more likely item. The fourth column of Table 1 contains the p-values for those subject-specific tests. Comparing the shaded and unshaded lines, we see a striking pattern. We cannot reject equality of the success rate and the expected success rate with $95 \%$ confidence for any of the high-success-rate subjects, and we reject equality with $90 \%$ confidence for only two of these subjects (and would have expected roughly one rejection by chance). In contrast, we reject equality at the $90 \%$ confidence level for seven of the eight low-success rate subjects (and with 88\% confidence for the eighth), at the 95\% confidence level for five, and at the 99\% confidence level for three. Visually, asterisks (indicating levels of statistical significance) tend to appear in the first data column when no asterisks appear in the fourth, and vice versa.

Overall, for high-success-rate subjects, the mean success rate is $68.2 \%$, while the expected success rate is $72.9 \%$; the difference (or bias) is modest but statistically significant $(p=0.042)$. Though the predictions are not right on the mark, they are remarkably close

\footnotetext{
32 Additional sources of subject-level variation in predictive success might include: local temperature variation during scanning, variability in the functioning of the imaging hardware, physiological noise (such as heart-rate variability), or subject motion. See Huettel et al. (2009), Chapter 8, for a discussion of noise sources in fMRI. We remove these factors when possible, but some (e.g. thermal and scanner-related noise) are difficult to measure and therefore to control.
} 
given the nature of our out-of-sample prediction exercise. Interestingly, our predictions are equally resolved for the low-success-rate subjects: the expected success rate is $72.6 \%$. However, the mean success rate for those subjects is only $53.5 \%$, and the difference (or bias) is large and highly statistically significant ( $\mathrm{p}<0.001)$.

One might be tempted to discount the preceding results as a possible coincidence: if the overall success rate is below the overall mean predicted probability, and if the latter does not vary between low- and high-success-rate subjects, then it is not surprising that the success rate for high-success-rate subjects is closer to that group's mean predicted probability of the more likely item. Thus, we view this first test as providing only a relatively weak preliminary indication concerning the model's performance among highsuccess-rate subjects.

Fortunately, a more demanding test is available. So far, we have made no use of variation in the strength of predictions across hold-out observations (e.g., whether the predicted probability of choosing the target item is $51 \%$ or $98 \%$ ). According to Table 1 , the mean within-subject standard deviation of the predicted probability is substantial $(0.140)$. Moreover, the predicted probability of the more likely item is distributed fairly evenly between $50 \%$ and $100 \%$ (see Figure A2 in Appendix A). Using this variation allows us to determine whether our predictive procedure is functioning properly. If, for example, the predicted probability averages 60\% within one large group of hold-out observations and $80 \%$ within a second group, and if the model is generating valid out-of-sample probabilities, the frequency with which the more likely item is chosen should be approximately $60 \%$ in the first group and approximately $80 \%$ in the second. Even if the model is just capturing tendencies, that frequency should be noticeably higher in the second group than in the first.

We implement this idea as follows. First, we rank the hold-out observations (pooled across all subjects) according to the predicted probability of the more likely choice (i.e., the probability of choosing the target item if the model indicates that the target is more likely, and the probability of choosing the non-target item if the model indicates that the nontarget item is more likely). Second, we divide the observations into deciles based on that probability. Third, for each decile, we compute the frequency with which the item identified as more likely was in fact chosen (i.e., the success frequency). Finally, we examine the 
relationship across deciles between the average predicted probability of choosing the more likely item and the actual frequency with which that item was actually chosen. ${ }^{33}$

Figure 2A plots the results, pooled over all subjects. The horizontal axis shows the predicted probability of choosing the more likely item, while the vertical axis shows the frequency with which that item was actually chosen. For an ideal predictive model, the data points would line up along the 45-degree line (i.e., the predicted probabilities and the success frequencies would always coincide). Though our procedure does not achieve this ideal, there is nevertheless an obvious and reasonably strong positive relationship between the predicted probabilities and success frequencies. Between the first and eighth deciles, the actual success rate increases roughly half a percentage point for every one percentage point increase in the predicted probability; beyond the eighth decile, it declines modestly. Overall, the predictive performance of the model is encouraging, at least directionally.

Figure 2B performs the same analysis separately for low- and high-success-rate subjects. The results are striking. For the eight low-success-rate subjects, there is no relationship between success frequencies and predicted probabilities: the line moves up and down a bit, but overall is fairly flat. With these problematic subjects removed, the procedure's performance is much improved. For the nine high-success-rate subjects, the relationship between success frequencies and predicted probabilities increases more sharply than the one in Figure 2A, and is much closer to the ideal (i.e., the 45 degree line). For the lowest two deciles, within which the average predicted probability is $53.8 \%$, the overall success frequency is $56.7 \%$, while for the highest two deciles, within which the average predicted probability is $92.7 \%$, the overall success frequency is $84.4 \%$.

To sharpen these impressions, we conduct additional statistical analyses. For each subject $i$ and choice trial $t$, we define a binary success indicator, $S_{i t}$, which equals unity when the subject chooses the item predicted as more likely (with this trial treated as a hold-out observation), and zero otherwise. Let $P_{i t}$ denote the predicted probability that the subject $i$ will choose the item identified as more likely in choice trial $t$ (again, when this choice trial is treated as a hold-out observation). Assuming that $P_{i t}$ is in fact a correct probability, it follows trivially that $\mathrm{E}\left[S_{i t} \mid P_{i t}\right]=P_{i t}$. Thus, $S_{i t}=P_{i t}+\varepsilon_{i t}$, where $\mathrm{E}\left[\varepsilon_{i t} \mid P_{i t}\right]=0$ (in particular, $\varepsilon_{i t}$ equals 1-P $P_{i t}$ with probability $P_{i t}$, and $-P_{i t}$ with probability $1-P_{i t}$ ). Accordingly, our strategy is to estimate simple linear probability models (LPMs) of the following form:

\footnotetext{
33 This procedure is motivated by and closely related to a goodness-of-fit test for binary choice models described by Lemeshow and Hosmer (1982).
} 


$$
S_{i t}=\alpha+\beta P_{i t}+\varepsilon_{i t} .
$$

If the predicted probability statements are in fact correct, we should obtain $\alpha=0$ and $\beta=1$.

We estimate two versions of the preceding linear probability models, one for the nine high-success-rate subjects, and one for the eight low-success-rate subjects. We use weighted least squares to account for the inherent heteroskedasticity in the linear probability models, following the procedure in Wooldridge (2003, page 455). In these regressions, each observation consists of a single hold-out choice pair; thus, the regression for high-success-rate subjects uses $50 \times 9=450$ observations, while the regression for lowsuccess-rate subjects uses $50 \times 8=400$ observations. For the eight low-success-rate subjects, we obtain an intercept of 0.551 (s.e. $=0.129$ ) and a slope of -0.023 (s.e. $=0.174$ ). The combination of low success rates and the absence of any detectable relationship between the two variables indicates that our forecasting procedure fails for those subjects. In contrast, for the nine high-success-rate subjects, we obtain an intercept to 0.118 (s.e. = 0.113 ) and a slope 0.775 (s.e. $=0.152$ ). Here, the relationship between the two variables is strong, positive, highly statistically significant, and within the general vicinity of the ideal. However, we reject the hypothesis that the intercept is in fact zero and the slope unity $(\mathrm{p}=$ 0.012). With that qualification, our prediction model performs well out of sample for the nine high-success-rate subjects.

Conceivably, the strong results obtained for the LPM estimated with high-successrate subjects could be attributable to compositional effects: success rates might be unrelated to predicted probabilities within subject, but subjects with higher success rates might also have higher predicted probabilities. In practice, Table 1 provides little reason to anticipate significant compositional effects, because the means and standard deviations of the predicted probabilities (the second and third data columns) are quite similar across subjects (the cross-subject standard-deviations of these statistics are only 0.028 in the case of the within-subject mean, and 0.010 in the case of the within-subject standard deviation).

To rule out the possibility that our LPM results for high-success-rate subjects reflect compositional effects, we estimate another LPM with subject-fixed effects. Our estimate of $\beta$ increases to 0.808 (s.e. $=0.157$ ). We also estimate an LPM separately for every subject. The slope coefficients and associated standard errors are reported in the last two data columns of Table 1 . Because each regression employs only 50 observations, the standard errors are large. Still, the overall pattern is striking. For the high-success-rate subjects, the slopes are all positive and range from a low of 0.133 to a high of 1.579 . The mean slope is 0.818 and 
the median is 0.955 , with three of the nine slopes exceeding unity. In contrast, for the lowsuccess-rate subjects, five of the eight slopes are negative. They range from a low of -0.420 to a high of 0.698 , with a mean of -0.002 and a median of -0.194 .

We conclude that our within-subject procedure for predicting choices involving new items performs successfully for roughly half (nine of seventeen) of our subjects. The overall success rate is $68 \%$ for that group, and subject-specific success rates are close to subjectspecific mean predicted probabilities of the more likely item, our expected success rates. Moreover, success frequencies mirror predicted probabilities across hold-out observations, both overall and within subjects. The predicted probabilities are not always spot-on for this group, but they are close.

We acknowledge that the procedure works poorly for the rest of our subjects: the overall success rate is only 54\%, subject-specific success rates differ considerably from subject-specific mean predicted probabilities, and success frequencies bear no discernable relation to predicted probabilities across hold-out observations. ${ }^{34}$

\section{B. Within-group predictions}

Our investigation in this subsection parallels that of Section 4.A, except that we study average behavior among groups, rather than the choices of specific individuals. Our objective is determine whether the average neural responses among a group of individuals contain enough information to make reasonably accurate predictions concerning the group's average behavior in new situations, using a model estimated with data concerning the same group.

\footnotetext{
34 An obvious question is whether there are any systematic and predictable differences between the subjects for whom the procedure works well and those for whom it works poorly. Although the experiment was not designed to address this question, we carried out the following three post-hoc exercises. First, we hypothesized that more attentive subjects might have higher success rates. However, we find no relationship between success rates and a subject's mean response time (RT) on catch trials, which is a proxy for attentiveness (Spearman's rho $-0.15, p=0.56$ for a test of the hypothesis of no correlation). Second, we hypothesized that it might be more difficult to predict choices for subjects who had weaker preferences across foods. The variance in their reported ratings is a proxy for the strength of their preferences. However, we found no relationship between this variance and success rates (Spearman's rho -0.10, $\mathrm{p}=0.70$ ). Finally, since head motion is a wellknown source of noise in fMRI studies, we investigated if this factor played a role. Standard fMRI preprocessing software computes 6 measures of head motion: shifts in the $\mathrm{x}, \mathrm{y}$, and $\mathrm{z}$ direction as well as the rotation measures pitch, roll, and yaw. Following common practice, we ignored rotation and dropped subjects whose head motion exceeded $2 \mathrm{~mm}$ in any direction in any of the six scanner runs. Among the remaining subjects, we found no relationship between motion and success rates (Spearman's rho -0.04, $\mathrm{p}=0.88$ ).
} 
Here we predict measures of subjective valuation, averaged across group members. A natural alternative strategy would have been to predict the fraction of subjects choosing the target item from a given pair. Unfortunately, that alternative is inconsistent with our experimental design, which employed different random pairings of the items for different subjects.

As explained in Section III, stage 3 of our experiment elicits preference ratings (on a scale of -3 to +3 ) for each item from every subject. We acknowledge that that our elicitation protocol is not incentive-compatible and that these ratings may not provide cardinally meaningful measures of willingness-to-pay (WTP), but we study them nevertheless for two reasons. First, preference ratings were elicited after the subjects made incentivized choices, from which it follows that (i) subjects had already thought about their preferences for each item in an incentive-compatible context, and (ii) subjects were likely to provide ratings that rationalized their choices. Second, these ratings were in fact highly correlated with choices: subjects choose the item with the highest rating $85.1 \%$ of the time in the 50 -choice condition (subjects 1-10; $\mathrm{p}<10^{-12}$, one-sided t-test vs. chance) and $90.1 \%$ of the time in the 200-choice condition (subjects 11-27: $\mathrm{p}<10^{-8}$, one-sided t-test vs. chance). Third, and more importantly, to the extent preference ratings are noisy measures of subjective valuation, our results likely understate the true predictive power of non-choice neural responses.

Statistical methods. Before aggregating subjective ratings across our 27 subjects, we normalized each subject's ratings using a z-score transformation. We then computed the mean normalized ratings for the group, denoted $Z_{j}$ for item $j$, as well as the group's mean non-choice neural responses, denoted $M_{j}$ for item $j$, where $M_{j}$ is a vector containing the average (across the group) neural response for each voxel $v$, denoted $M_{v j .}{ }^{35}$

As a first step, we simply ask whether the average non-choice neural responses to an item predict whether its average subjective rating is above or below the median rating (denoted $Z^{\text {med}}$ ). This is an interesting comparison because it stands in for a binary choice between the item in question and the median-rated alternative. We assume that the probability of an above-median rating for any item $j$ is given by the logistic function: ${ }^{36}$

$$
\operatorname{Pr}\left(Z_{j}>Z^{\text {med }} \mid M_{j}\right)=\frac{\exp \left(\gamma_{0}+\gamma M_{j}\right)}{1+\exp \left(\gamma_{0}+\gamma M_{j}\right)} .
$$

\footnotetext{
${ }^{35}$ See Figure A3 in Appendix A for the distribution of mean normalized ratings across food items.

36 The probability of any item falling above the median clearly depends on the entire vector of neural responses to all items. However, in our analysis, that vector is identical for all items (because all items are part of the same group); consequently, we suppress it in the notation.
} 
Plainly, realizations of this process cannot be independent across items (because half of the items must be above the median). However, with a sufficient number of items, correlations across observations are presumably small, so we ignore them and treat the model as a simple approximation of the true process.

By removing two items at a time from the set of 100, we create 50 training samples (each consisting of 98 observations) and 50 associated hold-out samples (each consisting of two observations). For each training sample, we then estimate the model and use it to predict whether the average valuations for the hold-out observations will fall above or below the median valuation of items within the training sample. We then assess the model's out-of-sample predictive performance over all 100 predictions. We classify a prediction as a success if the item's average subjective rating falls into the half of the training sample rating distribution that the model identifies as more likely.

As in the previous section, we applied a screening criterion to reduce the number of candidate voxels prior to estimating the model for any given training sample. Using only the training data, for each voxel $v$ we regressed $M_{v j}$ on a binary variable indicating whether $Z_{j}$ was above $Z^{\text {med. }}$. We then ranked the voxels according to the absolute values of the $\mathrm{t}$ statistics of the slope coefficients and retained those falling within some specified quantile. Then we estimated the probability model using the LASSO procedure, selecting the penalty parameter through 5-fold cross-validation, where the folds were assigned at random.

The second step in our analysis of group behavior was to predict the actual value of $Z_{j}$, an item's average subjective rating across all subjects, rather than a binary indicator of its position relative to the median. For this analysis, we employed a LASSO-penalized linear regression of $Z_{j}$ on $M_{j}$. In the initial screening step, for each voxel $v$ we regressed $M_{v j}$ on $Z_{j}$, then ranked all voxels by the t-statistics of the slope coefficients, and retained the highest $1 \%$. All other procedures were identical, except that the LASSO penalty parameter, $\lambda^{*}$, was chosen to maximize cross-validated mean-squared-error (which is appropriate here given that the objective is to predict a continuous variable).

As mentioned previously, the data gathered from our first 10 subjects are suitable for this analysis. Only the stage 2 choice data for those subjects have the feature that a single item plays a role in more than one observation (which produces violations of the assumed separation between training and hold-out samples), and we do not use those data here. Thus, throughout this section we present results based on all 27 subjects. 
Results. We begin with an analysis of predictions concerning the probability that the average subjective rating for a given hold-out item will fall above the median rating for items in the training sample. Figure 1 plots the overall success rate as a function of the percent of voxels retained after initial screening, with the retained percent ranging from $0.01 \%$ to $100 \%$. Our procedure maximizes the success rate when $0.5 \%$ of voxels are retained. The overall success rate is then 77\%, which represents an economically and statistically significant improvement over the uninformed 50\% benchmark $(p<0.001$, onesided t-test). Performance falls sharply when fewer than $0.5 \%$ of voxels are retained in the initial screening step, but is fairly robust when fewer are eliminated, with success rates generally exceeding $70 \%$. Recalling that classifications of ratings relative to the median stand in for binary choices between any given item and an alternative of median value, we note that we achieve a significantly higher overall success rate for within-group predictions than for the within-subject predictions discussed in Sections IV.A (compare the pertinent lines in Figure 1). To avoid cherry-picking results section-by-section, we adopt the same screening criterion here as in the previous section (1\%), which yields a success rate of $73 \%$, rather than the success-rate-maximizing $0.5 \%$ criterion. Our conclusions are not substantially affected by applying less restrictive screens.

Figure 3A illustrates the relationship between the predicted probability of an abovemedian rating and an item's average rating. Each data point corresponds to a food item; circles and crosses represent, respectively, correctly and incorrectly classified items. A strong positive relationship is easily discerned: our model plainly tends to predict higher probabilities of above-median ratings for more highly rated items.

As in Section IV.A, we perform an initial test of the validity of the model's predictive probability statements by comparing the average predicted probability with the overall success rate. On average, the model predicts that items will fall into the more likely half of the rating distribution with $79 \%$ probability. This figure is close to the actual success rate $(73 \%)$, and the gap is statistically insignificant ( $p=0.388$, two-sided t-test).

For a more discerning assessment of the model's predictive validity, we grouped items into quintiles (20 items in each) based on the predicted probability that the item's average rating exceeded the median, and then, for each quintile, computed the frequency with which the group's ratings of those items actually fell above the median. Results appear in Figure 3B. A strong positive relationship between predicted probabilities and realized 
frequencies is readily apparent. While the five data points do not line up along the 45 degree line, the empirical relation bears some resemblance to that ideal.

To sharpen this impression, we estimated a linear probability model (again using weighted least squares, to account for heteroskedasticity of the error term) relating a binary variable indicating whether an item's average rating was above the median to the predicted probability of that event. The estimated intercept is 0.173 (s.e. $=0.080$ ), and the slope is 0.624 (s.e. $=0.127$ ). We reject the joint hypothesis that the intercept is zero and the slope is unity with $95 \%$ confidence $(p=0.013)$. Although the point estimates may not support a literal interpretation of the model's predictive probability statements, on the whole its quantitative out-of-sample performance is promising.

Next we turn to predictions of the average rating itself, rather than its relation to the median. Figure 4 plots average normalized ratings against predicted ratings. The predictions are by no means exact, but there is once again a strong positive relationship. To summarize that relation, we regress the actual rating on the predicted rating using ordinary least squares and plot the regression line. With unbiased predictions, our regression would yield an intercept of zero and a slope of unity. We obtain an intercept of -0.012 (s.e. $=0.060$ ) and a slope of 0.712 (s.e. $=0.144$ ), and fail to reject the joint hypothesis of interest with $90 \%$ confidence $(p=0.136)$. The predicted ratings account for $20 \%$ of the variation in the actual ratings.

We conclude that our within-group procedure for predicting the average ratings of new items performs with considerable success. For the binary prediction task, the overall success rate is well over 70\%, considerably higher than for within-subject predictions, and predicted probabilities match up reasonably well with realized frequencies. Predicted ratings also track average ratings and plainly contain usefully predictive information.

Conceivably, one might achieve greater predictive accuracy by conditioning on higher moments of the distribution of predicted ratings. Likewise, it may be possible to predict additional parameters of the distribution of actual ratings, such as variance. These are important questions, but we leave them for future research.

\section{Predicting choices across groups}

The method of prediction developed and implemented in the previous section requires the use of separate forecasting models calibrated to each individual or group. If non-choice neural activity exhibits a sufficiently similar relation to choice across subjects, 
then it should be possible to construct a single prediction model and use it without recalibration to predict choices based on neural measurements taken from new individuals or groups. Such a model would have considerable practical value in that, once estimated, it would vastly simplify the steps required to formulate additional predictions. In particular, to predict behavior in new situations, one could collect data on non-choice neural responses to the relevant prospects for a new group of individuals, and apply the existing model. It would not be necessary to collect new measurements from the same set of individuals used to estimate the original model, or to re-estimate the model with additional data elicited from the new group. Indeed, with sufficient research, it might be possible to converge upon a single, stable formula for predicting new choices based on non-choice neural responses.

In this section we explore the feasibility of developing a single model for predicting choices from non-choice neural responses that is portable from one group to another. Specifically, we investigate whether it is possible to estimate the model with data on one group's choice distributions over various sets of items and, with reasonable accuracy, use it to predict another group's choice distributions over sets of new items.

Statistical methods. The methods used here are identical to those of Section VI.B, with some exceptions involving the nature of the training and hold-out samples. As in Section IV.B, all twenty-seven subjects were included in this analysis. Here, we randomly divide the subjects into a training group of 14 subjects and a hold-out group of 13 subjects. By removing two items at a time from the set of 100, we create 50 training sets (each consisting of 98 items) and 50 associated hold-out sets (each consisting of two items).

For each set of training items, we then estimate the same two models as in Section IV.B using data on the training subjects. We use one model to predict whether the average ratings of the hold-out subjects for the hold-out items will fall above or below the average rating of the median item for the hold-out subjects, and the other to predict the average ratings themselves.

To ensure that our results cannot be attributed to a potentially idiosyncratic division of the subjects, we repeat this exercise 200 times, selecting the training and holdout groups randomly each time. We thereby generate a total of 20,000 predictions.

Results and discussion. We begin with an analysis of predictions concerning the probability that the hold-out group's average subjective rating for a given hold-out item will fall above the median rating for items in the training data. Figure 1 plots the overall success rate (averaged over the 200 population draws) as a function of the percent of voxels 
retained after initial screening, with the retained percent ranging from $0.01 \%$ to $100 \%$ of voxels. Our procedure maximizes this rate when $50 \%$ of voxels are retained. The average overall success rate is then $61.2 \%,{ }^{37}$ which represents an economically and statistically significant improvement over the uninformed 50\% benchmark $(p<0.001$, one-sided t-test). Here, the initial voxel selection criterion has a fairly small effect on the success rate. To avoid cherry-picking results section-by-section, we will adopt the same screening criterion here as in Section IV (1\%), which yields an average overall success rate of $60.3 \%$, rather than the success-rate-maximizing 50\% criterion. Our conclusions are not substantially affected by applying less restrictive screens.

As in Section IV, we perform an initial check on the validity of the model's predictive probability statements by comparing the typical probabilistic prediction with the average overall success rate. On average, the procedure predicts that items will fall into the more likely half of the rating distribution with $79.7 \%$ probability. That figure is not close to the average overall success rate of $60.3 \%$, and the gap is statistically significant $(p<0.001$, twosided t-test). Consequently, the procedure does not generate quantitatively accurate probability statements for the hold-out data.

For a more revealing assessment of the model's predictive validity, we grouped individual predictions into deciles (2000 predictions in each) based on the predicted probability that the hold-out item's average rating among the hold-out group would exceed the median, and then, for each decile, computed the frequency with which the hold-out group's average ratings of those items actually fell above the median. Results appear in Figure 5, which shows a strong positive relationship between predicted probabilities and realized frequencies. The relationship does not, however, lie close to the 45 degree line.

To sharpen these impressions, we estimated linear probability models (via weighted least squares) relating a binary variable indicating whether the hold-out group's average rating of a hold-out item was above the median, to the predicted probability of that event. Pooling all 20,000 predictions, the estimated intercept is 0.317 ( $\mathrm{s}=0.006$ ), and the slope is 0.364 (s.e. $=0.010$ ). Adding fixed effects for each of the 200 population draws, the coefficient estimates and standard errors are the same to three decimal places. We also estimated a separate LPM for each population draw. The mean slope is 0.360 (s.e. $=0.158$ ), and the median is $0.354 .{ }^{38}$ Although these estimates do not support a literal interpretation

\footnotetext{
37 This figure represents the overall success rate averaged over the 200 population draws.

${ }^{38}$ Figure A4 in Appendix A shows the distribution of the resulting slope coefficients.
} 
of the model's predictive probability statements, they are directionally accurate. Thus, they provide evidence that the predicted probabilities contain a good deal of information that is useful for predicting across subjects.

As in our within-group exercise, we also directly predict the average rating using a linear regression with LASSO penalty. We then estimate an ordinary least squares regression of mean normalized rating (for the hold-out food in the hold-out group) on predicted rating for all 20,000 predictions, with fixed effects for each of the 200 population draws. The constant is -0.002 (s.e. 0.066) and the slope is 0.528 (se 0.012 ). The $\mathrm{R}^{2}$ from this regression is $0.091 .{ }^{39}$ While the results from this exercise are not as strong as for the withingroup analysis, the predicted ratings are clearly related to the actual ratings of the group.

\section{Some extensions}

In this section, we briefly summarize two extensions of our analysis. The first investigates whether it is possible to improve upon predictions derived with LASSO estimates through the use of alternative statistical tools. The second examines the anatomical location of predictive brain activity.

Zou and Hastie (2005) propose a procedure known as the Elastic Net, which they argue improves upon LASSO in many settings. The Elastic Net penalty is a convex combination of the LASSO $\left(\mathrm{L}_{1}\right)$ and Ridge $\left(\mathrm{L}_{2}\right)$ penalties. Like LASSO, it accomplishes variable selection, but has a greater tendency to retain correlated predictors (e.g., in the current context, activity in neighboring voxels). The procedure yields modest improvements. For example, with respect to the first prediction task examined in Section IV.B (predicting whether the average food rating for a group is above or below the median rating), the overall success rate is unchanged at 73\%. Notably, however, when we estimate a linear probability model relating an above-median indicator variable to the predicted probability that the item falls above the median, we obtain a slope coefficient that is close to unity $(0.870$, s.e. 0.171$)$; moreover, we fail to reject the joint hypothesis that the slope is one and the intercept $(0.064$, s.e. $=0.100)$ is zero $(p=0.692)$.

It is natural to wonder whether the predictive voxels are concentrated in regions that are known to play important roles in valuation (Rangel and Hare, 2010). Because

\footnotetext{
39 Figure A5 in Appendix A plots the mean normalized rating for each food, averaged over 200 population draws, versus the predicted rating for each food, again averaged over the population draws.
} 
LASSO retains only a small handful of predictors (21.1 on average in our analysis) and typically discards all but one of any highly correlated set, there is a tendency for the predictive voxels to be widely dispersed. That tendency is not necessarily bad from a predictive perspective. LASSO may benefit by selecting anatomically distant voxels with activity that is associated with the underlying value signal but that does not mirror localized noise, and indeed the Elastic Net, which in contrast tends to retain predictive clusters, performs only marginally better. However, the Elastic Net proves more useful in generating images of the anatomical locations of predictive voxels. For the Elastic Net estimates, we find that the predictive voxels are to a large extent concentrated in brain regions that are broadly associated with choice and value, including the ventral striatum, subgenual cingulate cortex, orbitofrontal cortex, insula, and inferior parietal lobe.

\section{Concluding remarks}

The preceding analysis points to the feasibility of inferring the choices people would make (if given the opportunity) at least in part based on their neural responses to prospects when they are not making actual decision making. It represents an important and challenging milestone in the process of developing methods for estimating choice mappings that could be used in settings where pertinent choice data are nonexistent, limited, or contaminated by spurious factors, so that more conventional methods of estimation are inapplicable or problematic. Possible examples include inferring willingness-to-pay for new products or for the avoidance of environmental damage, controlling for unobserved product characteristics in supply-and-demand estimation, and the estimation of the behavioral impact of interventions where naturally occurring events are insufficiently clean to permit reliable inferences.

It is important to acknowledge the limitations of our analysis. Our procedure is entirely unsuccessful for nearly half of our subjects. Moreover, even for subjects to whom it is applied successfully, in many instances it yields relatively weak predictions (e.g., predicted probabilities near 50 percent rather than 100\%), and consequently achieves only a moderate overall success rate (68.2\%).

We note, however, that our procedure also yields strong predictions in many instances. For the individual-level models, the degree of resolution is reasonably high overall, and the models are well-calibrated for just over half of our subjects. Compared with the successful half of our individual-level prediction exercises, our group-level prediction 
exercise achieves higher resolution, and the quality of calibration is comparable (though somewhat lower), despite the fact that we include all subjects, regardless of whether their individual-level prediction exercises were successful.

In addition, there is every reason to believe that refinements of the procedure will ultimately yield substantial improvements in predictive accuracy. Better methods can be developed to enhance attentiveness in the scanner and to weed out inattentive subjects. Advancements in knowledge of the brain and improved statistical methods may provide better guides to voxel selection. Technological advances will undoubtedly enhance our ability to detect and measure stimulus-specific neural responses.

Perhaps the greatest potential for improving predictive accuracy lies in exploring combinations of non-choice responses to potential prospects. One promising avenue is to supplement fMRI information with subjective non-choice responses, such as hypothetical choices, response times, and visual fixations, as well as other neurometric data, such as pupil dilation, ${ }^{40}$ facial temperature and muscle movement, SCRs, and the like. The latter types of measurements are easier and less costly to obtain than fMRI data, and may ultimately turn out to be highly predictive. Physiological responses may prove particularly valuable in detecting discrepancies between hypothetical statements and true tendencies.

\section{References}

Bateman, Ian J., Richard T. Carson, Brett Day, Michael Hanemann, Nick Hanley, Tannis Hett, Michael Jones-Lee, Graham Loomes, Susana Mourato, Ece Ozdemiroglu, David W. Pearce, Robert Sugden, and John Swanson, Economic Valuation with Stated Preference Techniques: A Manual, Edward Elgar: Northampton, 2002.

Belloni, A., Chernozhukov, V., and C. Hansen, "Inference Methods for High-Dimensional Sparse Econometric Models," Advances in Economics and Econometrics, 10 $0^{\text {th }}$ World Congress of the Econometric Society, 2011.

Bernheim, B. Douglas, "On the Potential of Neuroeconomics: A Critical (but Hopeful) Appraisal," American Economic Journal: Microeconomics, 1 (2009), 1-41.

Berns, Gregory S., C. Monica Capra, Sara Moore, and Charles Noussair, "Neural mechanisms of the influence of popularity on adolescent ratings of music," NeuroImage, 49 (2010), 26872696.

Blackburn, M., G. Harrison, and E. Rutstrom, "Statistical Bias Functions and Informative Hypothetical Surveys," American Journal of Agricultural Economics 76, 1994, 1084-88.

\footnotetext{
${ }^{40}$ See Kang et al. (2009) (response times) and Wang, Camerer, and Spezio (2010) (pupillometry).
} 
Blamey, R. K., J. W. Bennett, and M. D. Morrison, "Yea-Saying in Contingent Valuation Surveys," Land Economics, 75 (1999), 126-141.

Blumenschein, K., G. C. Blomquist, M. Johannesson, N. Horn, and P. Freeman P., "Eliciting willingness to pay without bias: evidence from a field experiment," Economic Journal 118, 2007, $114-137$.

Brier, G. W. , "Verification of forecasts expressed in terms of probability," Monthly Weather Review, 78 (1950), 1-3.

Brownstone, David, David S. Bunch, and Kenneth Train, "Joint mixed logit models of stated and revealed preferences for alternative-fuel vehicles," Transportation Research Part B: Methodological, 34 (2000), 315-338.

Camerer, C., G. Loewenstein, and D. Prelec, "Neuroeconomics: How neuroscience can inform economics," Journal of Economic Literature, 43 (2005), 9-64.

Champ, Patricia A., Richard C. Bishop, Thomas C. Brown, and Daniel W. McCollum, "Using Donation Mechanisms to Value Nonuse Benefits from Public Goods," Journal of Environmental Economics and Management, 33 (1997), 151-162.

Chua, Hannah Faye, S. Shaun Ho, Agnes J. Jasinska, Thad A. Polk, Robert C. Welsh, Israel Liberzon, and Victor J. Strecher, "Self-related neural response to tailored smoking-cessation messages predicts quitting," Nature Neuroscience, 14 (2011), 426-427.

Clithero, John A.; R. McKell Carter and Scott A. Huettel. 2009. "Local Pattern Classification Differentiates Processes of Economic Valuation." NeuroImage, 45(4), pp. 1329-38.

Clithero, John A.; David V. Smith; R. McKell Carter and Scott A. Huettel. 2011. "Within- and Cross-Participant Classifiers Reveal Different Neural Coding of Information." NeuroImage, 56(2), pp. 699-708.

Cramer, J., "Predictive Performance of the Binary Logit Model in Unbalanced Samples", The Statistician, 1999, 85-94.

Cummings, R. G., G. W. Harrison, and E. Rutstrom, "Homegrown values and hypothetical surveys: is the dichotomous choice approach incentive-compatible? American Economic Review 85, 1995, $260-266$.

Cummings, Ronald G., and Laura 0. Taylor, "Unbiased Value Estimates for Environmental Goods: A Cheap Talk Design for the Contingent Valuation Method," The American Economic Review, 89 (1999), 649-665.

Deichmann, R., J.A. Gottfried, C. Hutton, and R. Turner, "Optimized Epi for Fmri Studies of the Orbitofrontal Cortex," NeuroImage 19, 2001, 430-41.

Dijksterhuis, Ap, Maarten W. Bos, Loran F. Nordgren, and Rick B. van Baaren, "On Making the Right Choice: The Deliberation-Without-Attention Effect," Science, 311 (2006), 10051007.

Efron, Bradley. 1986. "How Biased Is the Apparent Error Rate of a Prediction Rule?" Journal of the American Statistical Association, 81(394), 461-70. 
Falk, Emily B., Elliot T. Berkman, Danielle Whalen, and Matthew D. Lieberman, "Neural activity during health messaging predicts reductions in smoking above and beyond selfreport," Health Psychology, 30 (2011), 177-185.

Fan, J., J. Lv, and L. Qi, "Sparse High-Dimensional Models in Economics," Annual Review of Economics 3, 2011, 291-317.

Fox, J.A., J.F. Shogren, D.J. Hayes, and J.B. Kliebenstein, "CVM-X: Calibrating Contingent Values with Experimental Auction Markets," American Journal of Agricultural Economics 80(3), 1998, 455-65.

Friedman, Jerome; Trevor Hastie and Rob Tibshirani. 2010. "Regularization Paths for Generalized Linear Models Via Coordinate Descent." Journal of statistical software, 33(1), 1.

Friston, Karl J., J. Ashburner, C. D. Frith, J. B. Poline, J. D. Heather, and R. S. J. Frackowiak, "Spatial Registration and Normalization of Images," Human Brain Mapping, 3(3), 1995, 16589.

Friston, Karl J., P. Fletcher, O. Josephs, A. Holmes, M. D. Rugg, and R. Turner, "Event-Related Fmri: Characterizing Differential Responses,” NeuroImage 7(1), 1998, 30-40.

Greene, William H., Econometric Analysis, Fifth Edition, Prentice Hall, 2003, 31-38.

Grosenick, L., S. Greer, and B. Knutson. "Interpretable Classifiers for Fmri Improve Prediction of Purchases." IEEE Transactions on Neural Systems and Rehabilitation Engineering, 16(6), 2008, pp. 539-48.

Gul, Faruk, and Wolfgang Pesendorfer, "The Case for Mindless Economics" in: The Foundations of Positive and Normative Economics, by Andrew Caplin and Andrew Shotter (eds.). Oxford University Press. 2008.

Hampton, A. N., and J. P. O'Doherty J, P., "Decoding the neural substrates of reward-related decision making with functional MRI," PNAS 104, 2007, 1377-1382.

Hare, T.A., J. O'Doherty, C.F. Camerer, W. Schultz, and A. Rangel, "Dissociating the role of the orbitofrontal cortex and the striatum in the computation of goal values and prediction errors," Journal of Neuroscience 28, 2008, 5623-5630.

Harrison, G., R. Beekman, L. Brown, L. Clements, T. McDaniel, S. Odom, and M. Williams, "Environmental Damage Assessment with Hypothetical Surveys: The Calibration Approach," Topics in Environmental Economics, M. Bowman, R. Brannlund, and B. Kristrim, eds., Amsterdam: Kluwer Academic Publishers, 1998.

Hastie, Trevor, Robert Tibshirani and Jerome Friedman. 2009. The Elements of Statistical Learning. New York: Springer.

Haxby, James V.; M. Ida Gobbini; Maura L. Furey; Alumit Ishai; Jennifer L. Schouten and Pietro Pietrini. 2001. "Distributed and Overlapping Representations of Faces and Objects in Ventral Temporal Cortex." Science, 293(5539), 2425-30. 
Haynes, John-Dylan, ed., 2011. "Multivariate Decoding and Brain Reading," Special isssue, NeuroImage, 56(2), 385-86.

Hsu, Ming, Meghana Bhatt, Ralph Adolphs, Daniel Tranel, and Colin F. Camerer, "Neural Systems Responding to Degrees of Uncertainty in Human Decision-Making," Science, 310 (2005), 1680-1683.

Huettel, Scott A.; Allen W. Song and Gregory McCarthy. 2009. Functional Magnetic Resonance Imaging. Sunderland, MA.

Jacquemet, Nicolas, Robert-Vincent Joule, Stephane Luchini, and Jason F. Shogren, "Preference Elicitation under Oath," working paper, 2011.

Johannesson, M., B. Liljas, P. O. Johansson, "An experimental comparison of dichotomous choice contingent valuation questions and real purchase decisions," Applied Economics 30, 1998, 643- 647.

Kable, Joseph W. and Paul W. Glimcher, 2007. "The Neural Correlates of Subjective Value During Intertemporal Choice," Nature Neuroscience, 10(12), 1625-33.

Kang, M. J., A. Rangel, M. Camus, and C. F. Camerer, 2009. "Hypothetical and real choice differentially activate common valuation areas," working paper.

Kang, M. J., A. Rangel, M. Camus, and C. F. Camerer, "Hypothetical and real choice differentially activate common valuation areas," Journal of Neuroscience 31, 2011, 461-468. Knutson, B., S. Rick , G. E. Wimmer, D. Prelec, and G. Loewenstein, "Neural predictors of purchases," Neuron 53, 2007, 147-156.

Kohavi, Ron. 1995. "A Study of Cross-Validation and Bootstrap for Accuracy Estimation and Model Selection," International joint Conference on artificial intelligence. Lawrence Erlbaum Associates Ltd, 1137-45.

Krajbich, Ian, Carrie Armel, and Antonio Rangel, "Visual fixations and the computation and comparison of value in simple choice," Nature Neuroscience 12(10), October 2010, 12921298.

Krajbich, Ian, Colin Camerer, John Ledyard, and Antonio Rangel, "Using neural measures of economic value to solve the public goods free-rider problem," Science 326, 2009, 596-599.

Kurz, Mordecai, "Experimental approach to the determination of the demand for public goods," Journal of Public Economics, 3 (1974), 329-348.

Lebreton, M., S. Jorge, V. Michel, B. Thirion, and M. Pessiglione, "An automatic valuation system in the human brain: evidence from functional neuroimaging," Neuron 64, 2009, 431439.

Lemeshow, Stanley, and David W. Hosmer, "A Review of Goodness of Fit Statistics for Use in the Development of Logistic Regression Models," American Journal of Epidemiology 115(1), 1982, 92-106. 
Levy, I., S. C. Lazzaro, R. B. Rutledge, and P. W. Glimcher, “Choice from non-choice: predicting consumer preferences from blood oxygenation level-dependent signals obtained during passive viewing," Journal of Neuroscience 31, 2011, 118-125.

Levy, I., J. Snell, A. J. Nelson, A. Rustichini and P. W. Glimcher, 2010. "Neural Representation of Subjective Value under Risk and Ambiguity." Journal of Neurophysiology, 103(2), 1036-47.

List, J. A., and C.A. Gallet, "What experimental protocols influence disparities between actual and hypothetical stated values?" Environmental Resource Economics 20, 2001, 241-254.

List, J.A., and J.S. Shogren, "Calibration of the difference between actual and hypothetical valuations in a field experiment," Journal of Economic Behavior and Organization 37, 1998, 193-205.

List, J.A., and J.S. Shogren, "Calibration of Willingness-to-Accept," Journal of Environmental Economics and Management 43, 2002, 219-33.

Litt, Ab; Hilke Plassmann; Baba Shiv and Antonio Rangel. 2011. "Dissociating Valuation and Saliency Signals During Decision-Making." Cerebral Cortex, 21(1), pp. 95-102.

Little, J., and R. Berrens, "Explaining disparities between actual and hypothetical stated values: further investigation using meta-analysis," Economic Bulletin 3, 2004, 1-13.

Loomis, John B., Kerri Traynor, and Thomas C. Brown, "Trichotomous Choice: A Possible Solution To Dual Response Objectives In Dichotomous Choice Contingent Valuation Questions," Journal of Agricultural and Resource Economics, 24 (1999), 572-583.

Mansfield, Carol, "A Consistent Method for Calibrating Contingent Value Survey Data," Southern Economic Journal, 64 (1998), 665-681.

Murphy, A. H. (1973). A New Vector Partition of the Probability Score. Journal of Applied Meteorology, 12(4), 595-600.

Murphy, J. J., P. G. Allen, T. H. Stevens, and D. Weatherhead, "A meta-analysis of hypothetical bias in stated preference valuation," Environmental Resource Economics 30, 2005, 313-325.

National Oceanic and Atmospheric Administration, "Natural resource damage assessment: Proposed rules”, Federal Register, 4 May, 59, 1994, 23098-23111.

Pereira, Francisco, Tom Mitchell and Matthew Botvinick. 2009. "Machine Learning Classifiers and fMRI: A Tutorial Overview." NeuroImage, 45(1, Supplement 1), pp. S199S209.

Plassmann, H., J. O’Doherty, and A. Rangel, “Orbitofrontal cortex encodes willingness to pay in everyday economic transactions," Journal of Neuroscience 27, 2007, 9984 -9988.

Plassmann, H., J. P. O’Doherty, and A. Rangel, “Appetitive and aversive goal values are encoded in the medial orbitofrontal cortex at the time of decision making," Journal of Neuroscience 30, 2010, $10799-10808$.

Rangel, A. and T. Hare, "Neural computation as associated with goal-directed choice," 
Current Opinion in Neurobiology, 2010, 20:1-9.

Ryali, Srikanth, Kaustubh Supekar, Daniel A. Abrams, and Vinod Menon, "Sparse Logistic Regression for Whole-Brain Classification of Fmri Data," NeuroImage 51(2), 2010, 752-64.

Shogren, J.F., "Experimental Markets and Environmental Policy," Agr. Res. Econ. Rev. 3, 1993, 117-29.

Shogren, J.F., "Experimental Methods and Valuation", in Handbook of Environmental Economics, K.-G. Maler and J.R. Vincent, Editors. 2005, Elsevier. p. 969-1027.

Shogren, J., "Valuation in the Lab," Environmental and Resource Economics, 2006. 34(1), 163 172.

Small, Kenneth A., Clifford Winston, and Jia Yan, "Uncovering the Distribution of Motorists' Preferences for Travel Time and Reliability," Econometrica, 73 (2005), 1367-1382.

Stone, Mervyn. 1974. "Cross-Validatory Choice and Assessment of Statistical Predictions." Journal of the Royal Statistical Society. Series B (Methodological), 111-47.

Tibshirani, Robert, "Regression Shrinkage and Selection Via the Lasso," Journal of the Royal Statistical Societ,. Series B (Methodological) 58(1), 1996, 267-88.

Tusche, A., S. Bode, and J. D. Haynes, "Neural responses to unattended products predict later consumer choices," Journal of Neuroscience 30, 2010, 8024-8031.

Tzourio-Mazoyer, N., B. Landeau, D. Papathanassiou, F. Crivello, O. Etard, N. Delcroix, B. Mazoyer, and M. Joliot, "Automated Anatomical Labeling of Activations in Spm Using a Macroscopic Anatomical Parcellation of the Mni Mri Single-Subject Brain," NeuroImage 15(1), 2002, 273-89.

Wang, J. T.-y., Spezio, M., \& Camerer, C. F. (2010). "Pinocchio's Pupil: Using Eyetracking and Pupil Dilation to Understand Truth Telling and Deception in Sender-Receiver Games," American Economic Review, 100(3), 984-1007

Wooldridge, Jeffrey M. 2003. Econometric Analysis of Cross Section and Panel Data.

Cambridge, MA: MIT Press Books.

Yates, J. Frank, "External correspondence: Decompositions of the mean probability score," Organizational Behavior and Human Performance, 30 (1982), 132-156.

Zou H., and T. Hastie (2005) "Regularization and variable selection via the elastic net." Journal of the Royal Statistical Society: Series B (Statistical Methodology) 67:301-320. 
Table 1. Predictive accuracy for choices involving new items, within subject.

\begin{tabular}{|c|c|c|c|c|c|c|}
\hline & & $\begin{array}{l}\text { Predict } \\
\text { of the } \mathrm{m}\end{array}$ & $\begin{array}{l}\text { orobability } \\
\text { likely iten }\end{array}$ & & & LPM \\
\hline Subject & Success rate & Mean & Std dev & $\begin{array}{c}p \text {-value for } \\
\text { bias }\end{array}$ & Slope & $\begin{array}{c}\text { Std err. of } \\
\text { slope }\end{array}$ \\
\hline 11 & $0.66^{* * *}$ & 0.663 & 0.138 & 0.966 & 0.966 & 0.485 \\
\hline 12 & 0.52 & 0.702 & 0.156 & $0.013^{* *}$ & 0.550 & 0.459 \\
\hline 13 & $0.62^{* *}$ & 0.728 & 0.143 & $0.090^{*}$ & 1.579 & 0.434 \\
\hline 14 & $0.66^{* * *}$ & 0.768 & 0.133 & 0.126 & 0.133 & 0.517 \\
\hline 15 & 0.58 & 0.711 & 0.128 & $0.074^{*}$ & 0.230 & 0.559 \\
\hline 16 & 0.52 & 0.742 & 0.147 & $0.005^{* * *}$ & -0.075 & 0.494 \\
\hline 17 & 0.58 & 0.730 & 0.136 & $0.050^{*}$ & -0.325 & 0.527 \\
\hline 18 & $0.76^{* * *}$ & 0.738 & 0.141 & 0.726 & 0.393 & 0.437 \\
\hline 19 & 0.54 & 0.733 & 0.142 & $0.014^{* *}$ & -0.332 & 0.508 \\
\hline 20 & 0.58 & 0.692 & 0.148 & 0.114 & 0.698 & 0.476 \\
\hline 21 & $0.62^{* *}$ & 0.748 & 0.140 & $0.070^{*}$ & 0.517 & 0.499 \\
\hline 22 & $0.72^{* * *}$ & 0.697 & 0.130 & 0.727 & 0.381 & 0.501 \\
\hline 23 & $0.68^{* * *}$ & 0.717 & 0.144 & 0.567 & 0.955 & 0.454 \\
\hline 24 & $0.70^{* * *}$ & 0.763 & 0.118 & 0.320 & 1.328 & 0.544 \\
\hline 25 & 0.44 & 0.760 & 0.145 & $0.000^{* * *}$ & -0.420 & 0.494 \\
\hline 26 & 0.52 & 0.733 & 0.141 & $0.007^{* * *}$ & -0.312 & 0.515 \\
\hline 27 & $0.72^{* * *}$ & 0.738 & 0.155 & 0.761 & 1.114 & 0.392 \\
\hline Group & & & & & & \\
\hline Mean & $0.613^{* * *}$ & 0.727 & 0.140 & $<0.001^{* * *}$ & 0.434 & 0.488 \\
\hline Std Dev & 0.089 & 0.028 & 0.010 & & 0.615 & 0.043 \\
\hline
\end{tabular}

NOTES: Based on an initial voxel selection threshold of 0.01 . Asterisks are used to denote statistical significance only in the columns for "success rate" (difference from uninformed benchmark of 0.50 , binomial test for individual rates, 1-sided t-test for group mean rate) and "p-value for bias" (difference between success rate and mean predicted probability, two-sided t-test), as follows: * denotes $\mathrm{p}<0.1$; ** denotes $\mathrm{p}<0.05 ; * * *$ denotes $\mathrm{p}<0.01$. "Success rate" is the frequency with which the item with highest predicted choice probability in each pair was actually chosen; "p-value for bias" refers to the test statistic for the hypothesis that the success rate equals the mean predicted probability, and "LPM" refers to a simple linear probability model relating a success indicator to the predicted probability. "Group Mean" is the mean of within-subject means, and "Std Dev" is the standard deviation of within-subject means. 
Figure 1. Overall success rate as a function of the percent of voxels retained after initial screening when predicting choices for new items. The between group standard errors are bootstrapped using the 200 population draws.

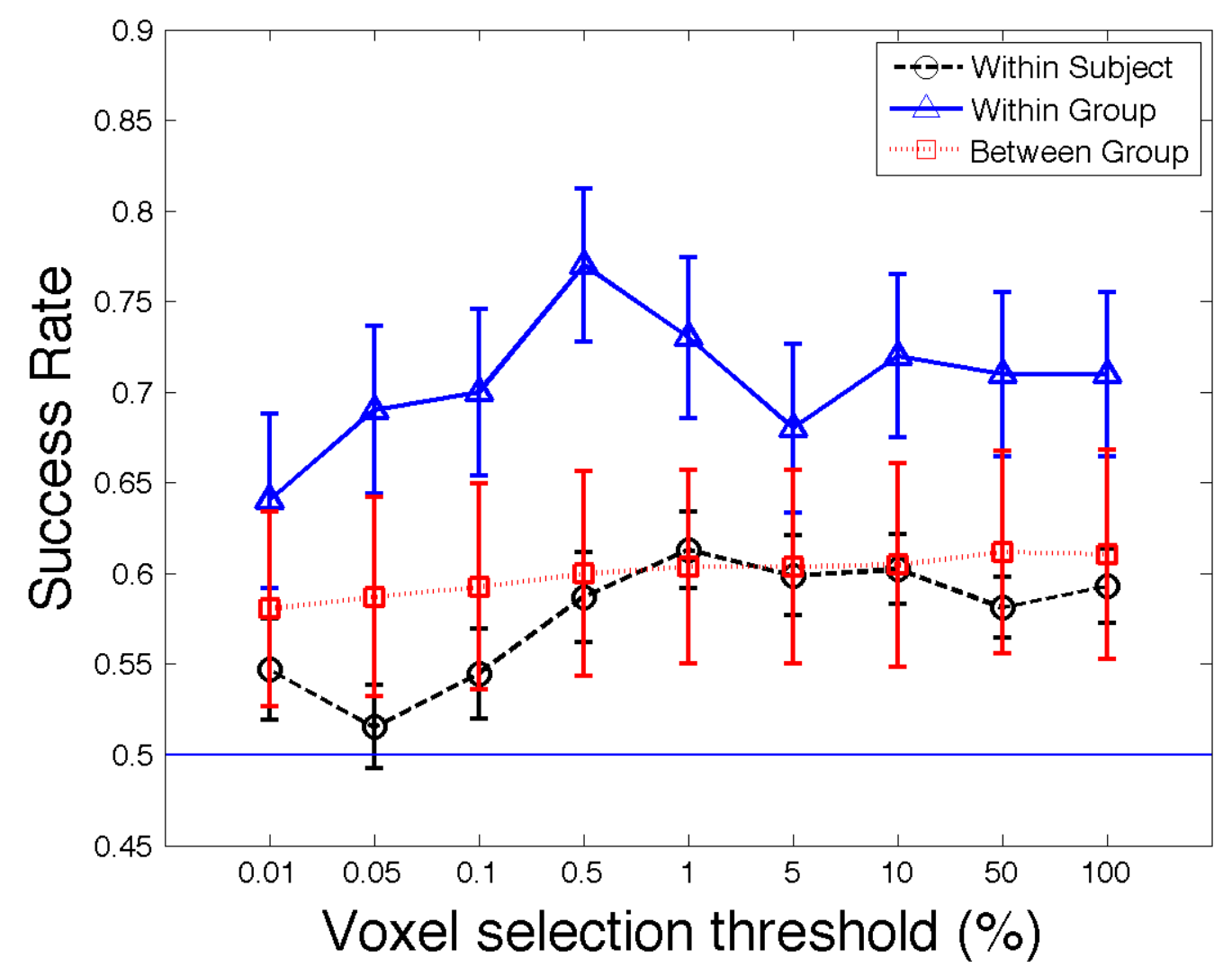


Figure 2. Success rate for within-subject predictions of choices involving new items as a function of predictive choice probability of the more likely item for (A) the entire group, and (B) separately for high-success-rate and low-success-rate subjects.

A

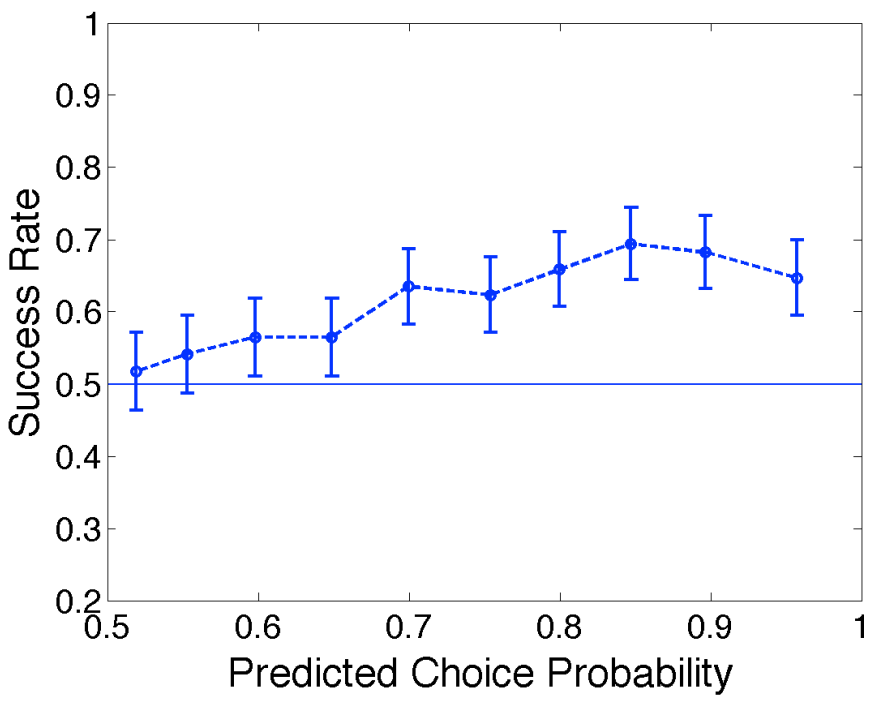

B

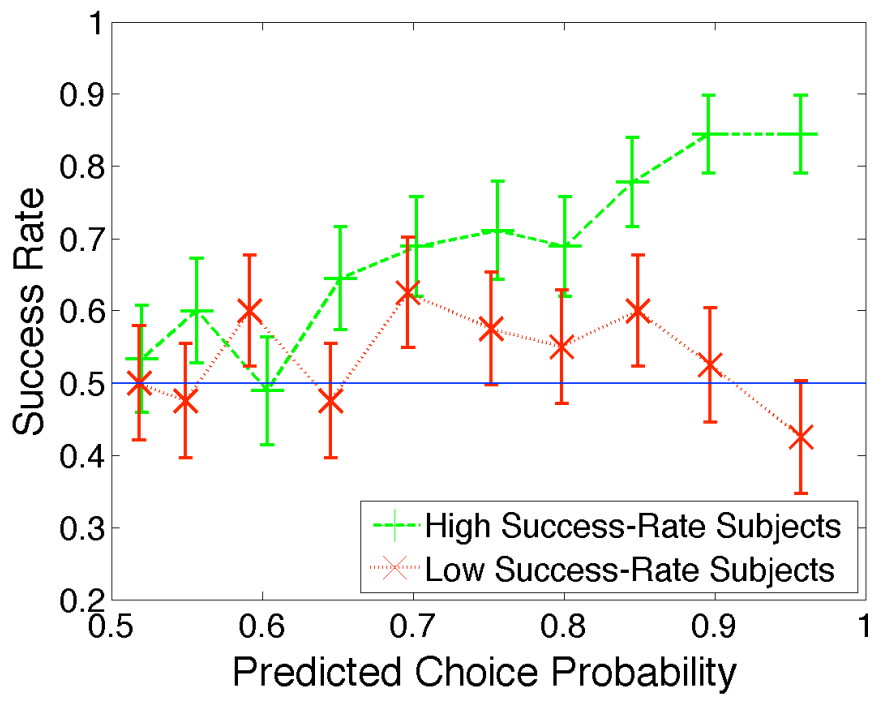


Figure 3. Predicting above-and-below-median ratings for new items within groups. (A) Scatter plot of mean ratings versus predicted probability that item is in the upper half of the group's valuation distribution. Circles denote correct predictions. Crosses denote incorrect predictions. (B) Fraction of items with ratings exceeding the median versus average predicted probability of rating exceeding the median, grouped by quintiles of the latter.

A

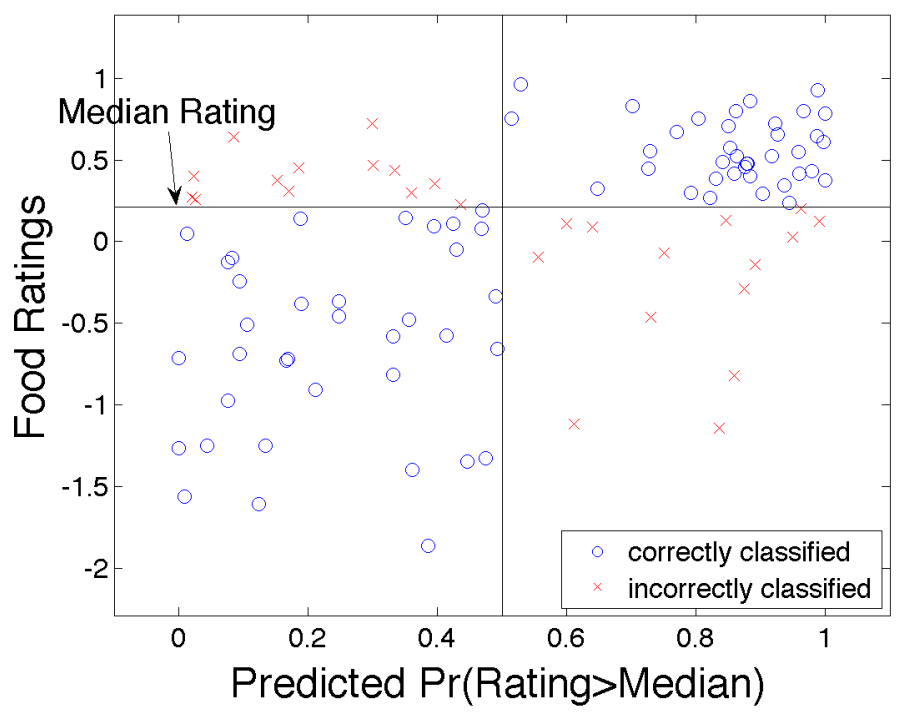

B

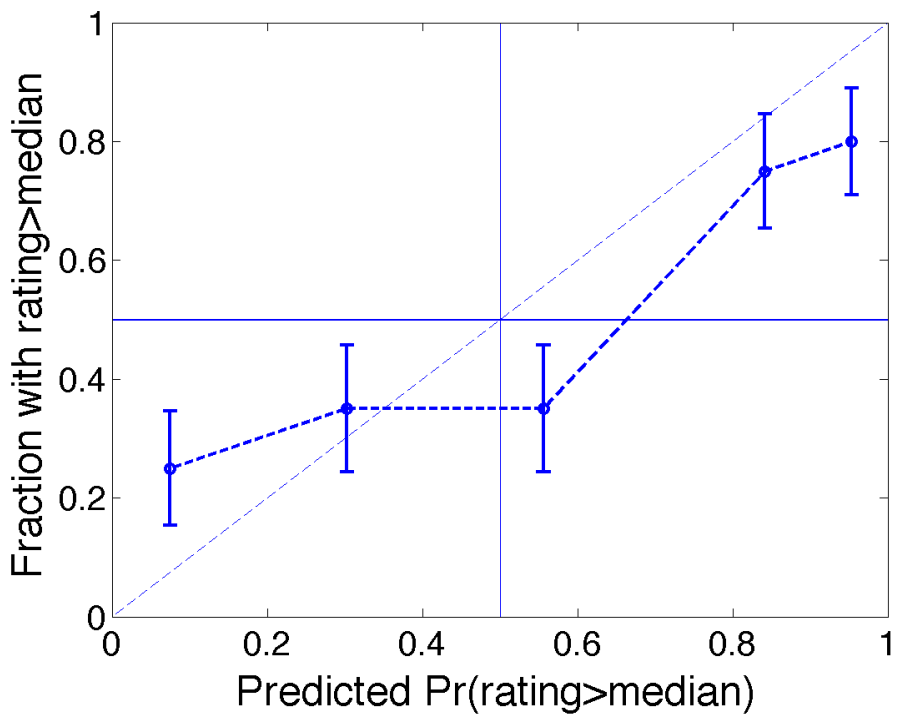


Figure 4. Predicting average ratings for new items within groups. Scatter plot of actual vs. predicted mean normalized ratings for each item. Each point represents a different food item. Least-squares regression line included.

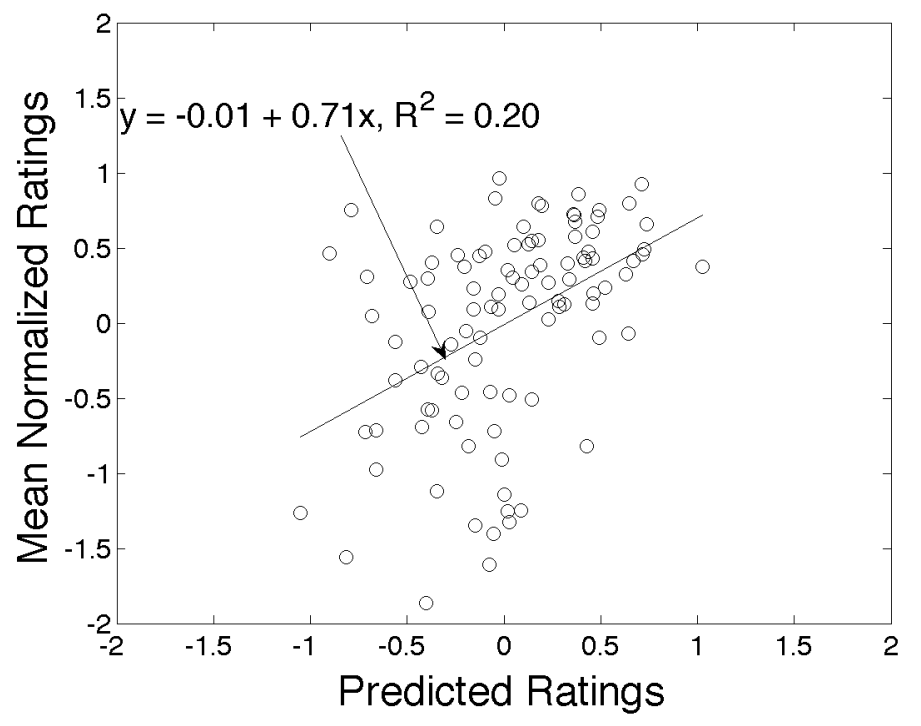

Figure 5. Predicting above-and-below-median average ratings for new items and new groups. Fraction of items with ratings exceeding the median versus average predicted probability of rating exceeding the median, grouped by deciles of the latter. Standard errors computed via bootstrap over the 200 population draws.

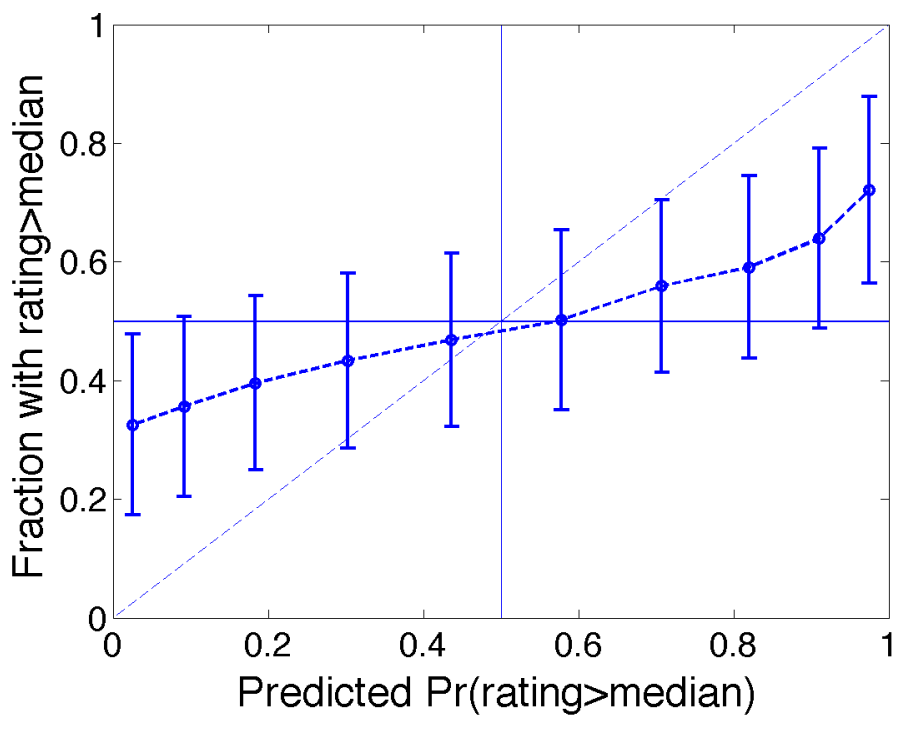

